\title{
Disturbed Processing of Contextual Information in HCN3 Channel Deficient Mice
}

\begin{abstract}
Marc S. Stieglitz ${ }^{1}$, Stefanie Fenske ${ }^{1}$, Verena Hammelmann ${ }^{1}$, Elvir Becirovic ${ }^{1}$, Verena Schöttle ${ }^{1}$, James E. Delorme ${ }^{2}$, Martha Schöll-Weidinger ${ }^{1}$, Robert Mader ${ }^{1}$, Jan Deussing ${ }^{3}$, David P. Wolfer ${ }^{4,5,6}$, Mathias W. Seeliger ${ }^{7}$, Urs Albrecht ${ }^{2}$, Carsten T. Wotjak ${ }^{3}$, Martin Biel ${ }^{1}$, Stylianos Michalakis ${ }^{1}$ and Christian Wahl-Schott ${ }^{1 *}$

${ }^{1}$ Center for Integrated Protein Science and Center for Drug Research, Department of Pharmacy, Ludwig-Maximilians University, Munich, Germany, ${ }^{2}$ Neurobiochemistry of Circadian Rhythms, Department of Biology, University of Fribourg, Fribourg, Switzerland, ${ }^{3}$ Department of Stress Neurobiology and Neurogenetics, Max Planck Institute of Psychiatry, Munich, Germany, ${ }^{4}$ Institute of Anatomy, University of Zurich, Zurich, Switzerland, ${ }^{5}$ Department of Health Sciences and Technology, Institute of Human Movement Sciences and Sport, ETH Zurich, Zurich, Switzerland, ${ }^{6}$ Neuroscience Center Zurich, University of Zurich and ETH Zurich, Zurich, Switzerland, ${ }^{7}$ Ocular Neurodegeneration Research Group, Centre for Ophthalmology, Institute for Ophthalmic Research, Eberhard Karls University Tuebingen, Tuebingen, Germany
\end{abstract}

Hyperpolarization-activated cyclic nucleotide-gated channels (HCNs) in the nervous system are implicated in a variety of neuronal functions including learning and memory, regulation of vigilance states and pain. Dysfunctions or genetic loss of these channels have been shown to cause human diseases such as epilepsy, depression, schizophrenia, and Parkinson's disease. The physiological functions of HCN1 and HCN2 channels in the nervous system have been analyzed using genetic knockout mouse models. By contrast, there are no such genetic studies for HCN3 channels so far. Here, we use a $\mathrm{HCN} 3-$ deficient $\left(\mathrm{HCN}^{-/-}\right)$mouse line, which has been previously generated in our group to examine the expression and function of this channel in the CNS. Specifically, we investigate the role of $\mathrm{HCN} 3$ channels for the regulation of circadian rhythm and for the determination of behavior. Contrary to previous suggestions we find that $\mathrm{HCN}^{-/-}$mice show normal visual, photic, and non-photic circadian function. In addition, $\mathrm{HCN}^{-/-}$ mice are impaired in processing contextual information, which is characterized by attenuated long-term extinction of contextual fear and increased fear to a neutral context upon repeated exposure.

Keywords: HCN3, HCN channel, circadian rhythm, fear memory, contextual information, intergeniculate leaflet, behavior mouse, knockout mouse

\section{INTRODUCTION}

Hyperpolarization-activated cyclic nucleotide-gated channels are widely expressed in the brain and other parts of the central and peripheral nervous systems (Pape, 1996; Robinson and Siegelbaum, 2003; Biel et al., 2009). They are particularly involved in the spatial (Nolan et al., 2004; Giocomo et al., 2011; Hussaini et al., 2011) and working memory (Wang et al., 2007), cerebellum dependent motor learning (Nolan et al., 2003), thalamic control of vigilance states (McCormick and Bal, 1997) and neuropathic pain (Emery et al., 2011). Owing to the distinct properties of these channels, different degrees of dysfunction up to a complete loss of function lead to neurological and 
psychiatric disorders such as epilepsy (Shah et al., 2004, 2013; Baruscotti et al., 2010; Reid et al., 2012), depression (Kim et al., 2012), and schizophrenia (Yi et al., 2016). HCN channels belong to the superfamily of voltage-gated pore loop cation channels. While they are principally operated by hyperpolarization, they are also tightly regulated by hormones and neurotransmitters that act via the second messengers cyclic AMP and cyclic GMP. The HCN channel family comprises four highly homologous members ( $\mathrm{HCN} 1$ through $\mathrm{HCN} 4)$. All four isoforms have been identified in the central nervous system (CNS). The current produced by these channels, termed $\mathrm{I}_{\mathrm{h}}$, plays a fundamental role in controlling excitability and other electric properties of cells. In brain, $\mathrm{I}_{\mathrm{h}}$ has been shown to play a key role in the control of basic functions of neurons, including determination of resting membrane potential, dendritic integration, synaptic transmission, and action potential (AP) firing (Biel et al., 2009). These functions have been attributed almost exclusively to $\mathrm{HCN} 1, \mathrm{HCN} 2$, and $\mathrm{HCN} 4$ channels. Interestingly, much less is known about the function of $\mathrm{HCN} 3$ channels in the CNS. The distribution of HCN3 protein in the CNS has been systematically analyzed using immunohistochemical studies in the rat where $\mathrm{HCN} 3$ expression was found in several brain regions including nuclei of the amygdala, paraventriular nucleus, olfactory bulb, lateral hypothalamic area, and others (Notomi and Shigemoto, 2004). So far, a few studies have attempted to elucidate functional aspects of these channels (Arroyo et al., 2006; Kanyshkova et al., 2009, 2012; Battefeld et al., 2012; Kretschmannova et al., 2012; Leist et al., 2016). Notably, in the mouse, a study postulated that HCN3 channels might be involved in the regulation of the circadian system (Ying et al., 2011). Specifically, it has been reported that HCN3 channels are present in the intergeniculate leaflet (IGL) of the hypothalamus (Notomi and Shigemoto, 2004; Ying et al., 2011). This nucleus is considered to be critical for the integration and transmission of entrainment cues to the suprachiasmatic nucleus (SCN) (Figure 1). However, no functional behavioral data have been published which investigated the role of HCN3 in the circadian system. Furthermore, expression of HCN3 has been reported in the retina (Muller et al., 2003). However, functional evidence in favor of a physiological role of $\mathrm{HCN} 3$ in vision is also lacking so far.

In the present study, we made use of a HCN3-deficient mouse line to experimentally address the question to which extent HCN3 channels are involved in the regulation of circadian rhythm. In addition, we explored whether $\mathrm{HCN} 3$ channels in the brain are determining factors for behavior.

\section{MATERIALS AND METHODS}

\section{Ethical Statement}

The studies were carried out in accordance with the approved guidelines of the local committee of laboratory animal care (District Government of Upper Bavaria; Experiment allowance number 55.2-1-54-2531-88-05) and German Laws on animal welfare (Tierschutzgesetz). All procedures concerning animals adhered to the ARVO statement for the Use of Animals in Ophthalmic and Vision Research and the procedures were performed with permission of the competent government authority (Regierungspraesidium Tuebingen). Circadian experiments were done according to the law for animal protection authorized by the veterinary office of the Canton of Fribourg, Switzerland.

\section{Animals and Housing}

For the present study we used the previously published knockout mouse line, $\mathrm{HCN}^{-/-}$, in which $\mathrm{HCN} 3$ is globally deleted (Fenske et al., 2011). This mouse line was generated using Cre/loxP-based deletion of exon 2, which encodes the first four transmembrane helices of the channel (for details see Fenske et al., 2011). After establishing the new knockout mouse line, the animals were backcrossed from a mixed C57BL6/129SV background to C57BL6/N animals for five generations before the progeny was used for testing. For this study, all experiments were performed on global $\mathrm{HCN3}^{-/-}$mice and their wild type littermates whose genotypes were confirmed by PCR experiments. Unless indicated, animals were housed under an inverse $12 \mathrm{~h}$ light/dark cycle where lights were switched off at $7 \mathrm{a} . \mathrm{m}$. and animals hat ad-libitum access to food and water.

\section{Immunohistochemistry}

Twelve micrometer thick coronal cryosections of adult mouse brain were fixed with $4 \%$ paraformaldehyde in PBS and blocked with PBS containing 10\% normal chicken serum (Vector laboratories) and $0.3 \%$ Triton X-100. Primary and secondary antibodies and dilutions used are listed below. Incubation with primary antibodies was done overnight at $4^{\circ} \mathrm{C}$, and with secondary antibodies $2 \mathrm{~h}$ at room temperature. In experiments using horseradish peroxidase-conjugated (HRP) secondary antibodies ( $\mathrm{HCN}$ channels), endogenous peroxidase activity was quenched $\left(3 \% \mathrm{H}_{2} \mathrm{O}_{2}\right.$ in methanol) before sections were incubated with HRP antibodies. In these experiments, tyramide signal amplification was performed according to manufacturer's instruction using Cy3-conjugated tyramide (TSA-Plus Cyanine 3 System, Perkin Elmer). To visualize cell nuclei, slices were counter stained with $5 \mu \mathrm{g} / \mathrm{ml}$ Hoechst 33342. The brain slices were examined on an epifluorescence microscope (Axioplan 2, Zeiss) and a confocal laser scanning microscope (LSM 510, Zeiss).

\section{Western Blot}

Electrophoretic separation of proteins was done by a SDSPAGE. Electrophoresis was performed in a mini gel apparatus (Protean 3, Biorad) at $100 \mathrm{~V}$, before the gel was transferred to a tank-blot-system (Mini Trans blot, Biorad) onto a PVDF membrane (Immobilon, Millipore). After blotting $(1 \mathrm{~h}, 100 \mathrm{~V})$ the membrane was dried and then blocked with 5\% milk powder/TBS for $1.5 \mathrm{~h}$. Primary and secondary antibodies and dilutions used are listed below. Incubation with primary antibody was done overnight at $4^{\circ} \mathrm{C}$. For detection, a HRP based ECL Kit (ECL system, Amersham) was used. Therefore, the membrane was incubated with HRP conjugated secondary antibody (HRP, Jackson) at RT for $1 \mathrm{~h}$. The exposed films (Hyperfilm ECL, Amersham) were developed by a Curix 60 (Agfa). 


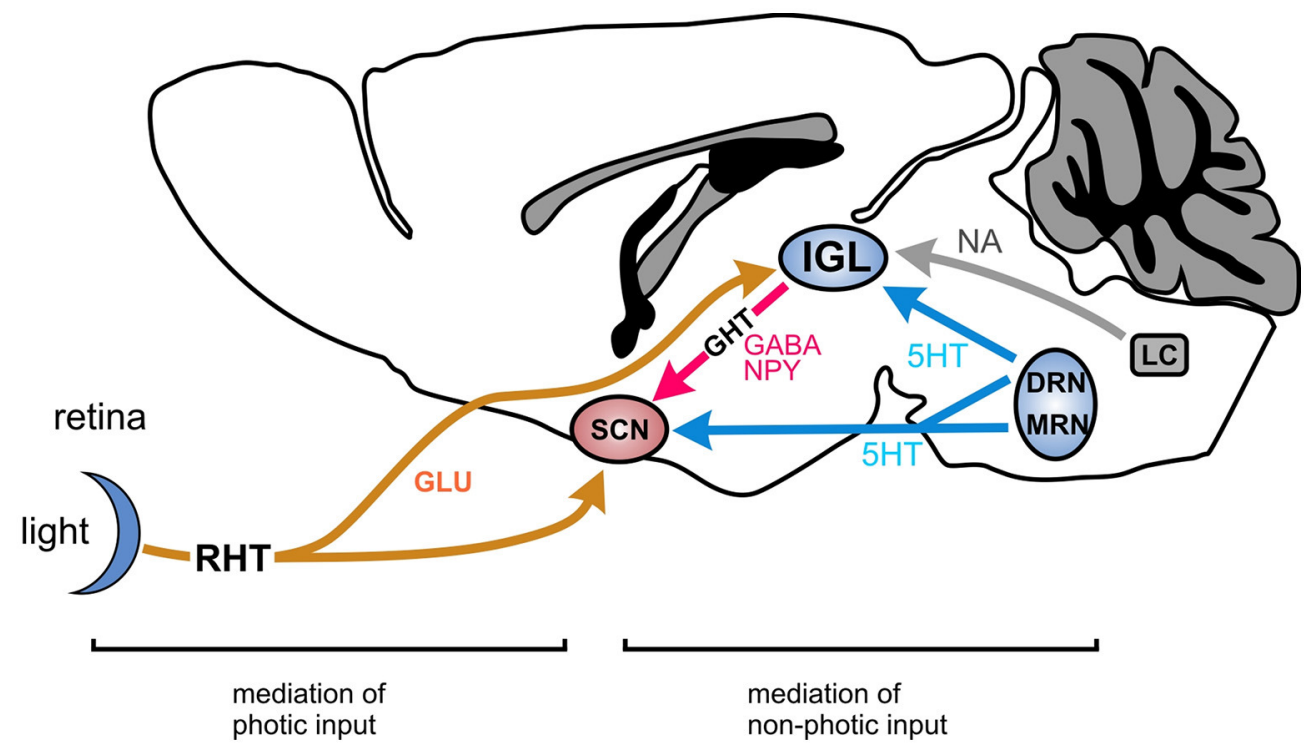

FIGURE 1 | Main pathway of the circadian system in mammals involving the SCN and the IGL. The principal pathway for photic entrainment is the retinohypothalamic tract $(\mathrm{RHT})$, a glutamatergic projection from the retina to the suprachiasmatic nucleus (SCN), and the intergeniculate leaflet (IGL). Non-photic entrainment cues converge on the IGL from the dorsal raphe nuclei (DRN) and the locus coeruleus (LC) and other nuclei involved in the regulation of sleep, arousal and activity. The IGL integrates these photic and non-photic inputs and conveys this information to the SCN via the geniculohypothalamic tract (GHT) which is a GABA-NPY projection. GLU, glutamate; GABA, gamma-aminobutyric acid; NPY, neuropeptide Y; $5 \mathrm{HT}$, serotonin; MRN, mediane raphe nucleus.

Antibodies Used

\section{Primary Antibodies}

Immunohistochemistry

Antibody Dilution Manufacturer

rb anti-HCN3 1:1000

rb anti-HCN1 1:1000

rb anti-HCN2 1:1000

rb anti-HCN4 1:500

(Mistrik et al., 2005), \#9495/1

Alamone, APC-030

Alamone, APC-056

Alamone, APC-052

Western blot

rb anti-HCN3 1:2000 (Mistrik et al., 2005), \#9495/1

ms anti-Tubulin 1:400 Dianova, MS-719-P1

\section{Secondary Antibodies \\ Immunohistochemistry \\ Antibody Dilution Manufacturer \\ dk anti-rb HRP 1:1000 Jackson \\ dk anti-gp FITC 1:200 Jackson}

Western blot

dk anti-rb HRP 1:2000 Amersham

$\mathrm{dk}$ anti-ms HRP 1:2000 Amersham

\section{Preabsorption of HCN3 Antibody}

Unspecific binding of the HCN3 antibody was avoided by preabsorption of the antibody against $\mathrm{HCN}^{-/-}$tissue. An adult $\mathrm{HCN}^{-/-}$mouse was anesthetized and decapitated and its brain was removed, reduced to small pieces with a scalpel, and stored in a $1.5 \mathrm{~mL}$ Eppendorf tube. One milliliter of protease inhibitor cocktail was added and the tissue was homogenized on ice using the Ultra Turrax (T8.01, IKA-works). Ten microliters of \#9495/1
HCN3-antibody were added and the mixture was agitated in an overhead shaker at $4{ }^{\circ} \mathrm{C}$ for $2.5 \mathrm{~h}$. Afterwards, the mixture was centrifuged at $13,400 \mathrm{rpm}$ for $15 \mathrm{~min}$ at $4^{\circ} \mathrm{C}$, the supernatant was collected into an ultracentrifugation tube and centrifuged at $30,000 \mathrm{rpm}$ for $45 \mathrm{~min}$. Then, sodium-azide was added to a final concentration of $0.05 \%$ and the preabsorbed antibody was stored on $4^{\circ} \mathrm{C}$ until usage.

\section{Determination of Standard Laboratory Blood Plasma Parameters}

For measurements of the blood plasma parameters 10 wild type and $11 \mathrm{HCN}^{-/-}$animals were used. The mice were restrained and blood samples were collected from the lateral tail vein into lithium heparin-coated Microvette tubes (Sarstedt). Blood samples were centrifuged for $10 \mathrm{~min}$ at 3,000 rpm and $4^{\circ} \mathrm{C}$, and $150 \mu \mathrm{l}$ of plasma were stored on $-20^{\circ} \mathrm{C}$ until investigation. Plasma parameters were analyzed with a Hitachi 717 autoanalyzer and reagents from Roche after diluting the plasma samples 1:2 with $\mathrm{H}_{2} \mathrm{O}$ as previously described (Rathkolb et al., 2000).

\section{Determination of Corticosterone Levels}

The blood corticosterone levels of $10 \mathrm{WT}$ and $10 \mathrm{HCN}^{-/-}$ animals were determined from animals housed under a standard 12/12h light-dark cycle where lights were switched on at 7 a.m. At 7 a.m., 1 p.m., and 7 p.m. [zeitgeber time (ZT) 0, ZT 6, and ZT 12 , respectively]. One animal had to be excluded due to failure of the radioimmunoassay (RIA). Animals were anesthetized using Isoflurane $\left(5 \mathrm{l} / \mathrm{min}\right.$ in $95 \% \mathrm{O}_{2}$ and $\left.5 \% \mathrm{CO}_{2}\right)$ and retrobulbar blood samples were taken using heparin-coated test tubes (Kabe 
Labortechnik $\mathrm{GmbH}$ ). By centrifugation (10 min, 8,500 rpm, RT) the serum was separated and stored at $-80^{\circ} \mathrm{C}$ until further processing.

\section{Corticosterone RIA}

A commercially available RIA kit (ImmunoChem Double antibody Corticosterone ${ }^{125}$ J RIA Kit, 07-120103, MP Biomedicals for Corticosterone) was used according to manufacturer's protocol. The sensitivity of the assay is $7.7 \mathrm{ng} / \mathrm{mL}$ corticosterone and the variation was measured to be $4.4 \%$ between samples and $6.5 \%$ between runs.

\section{CRF Concentration Determination}

For this experiment animals were housed under standard $12 / 12 \mathrm{~h}$ light-dark conditions with lights switched on at 7 a.m. CRF concentrations in the brain of $10 \mathrm{WT}$ and $10 \mathrm{HCN}^{-/-}$animals were determined in two groups at either 7 a.m. (ZT 0) or 7 p.m. (ZT 12). Therefore, mice were anesthetized and their whole brains - excluding the olfactory bulb - were removed and homogenized on ice in $4 \mathrm{~mL}$ lysis buffer using Ultra Turrax (type T8.01, IKA-Werke). Samples were centrifuged (Optima LE-80K ultracentrifuge, Beckmann Coulter) with 15,000 rpm for $20 \mathrm{~min}$ at RT. Then, the samples were flash-frozen on liquid nitrogen and stored on $-80^{\circ} \mathrm{C}$ until further processing.

\section{CRF-Elisa}

Commercially available ELISA kit (YII-YK130-EX, Cosmo Bio Co., Ltd.) was used according to manufacturer's instruction. Fluorescence was detected using a photometric optical reader (Opsys MR, Thermo Labsystems) at $490 \mathrm{~nm}$. CRF contents were calculated using a standard curve $(0-10 \mathrm{ng} / \mathrm{mL})$. The sensitivity of the essay is $0.313 \mathrm{ng} / \mathrm{mL}$ for CRF. The intra-assay variation coefficient was $2.72-7.11 \%$ and inter-assay variation coefficient was $5.01-9.40 \%$.

\section{Assessment of Bodyweight}

After weening, WT $(n=10)$ and $\mathrm{HCN}^{-/-}(n=10)$ animals received water and food (standard laboratory animal chow; ssniff $\mathrm{R} / \mathrm{M}-\mathrm{H}$ ) ad-libitum and their bodyweight was determined at the age of 12 weeks.

\section{Electrophysiology}

\section{Brain Slice Preparation}

Brain slice preparation was performed as previously described elsewhere, with slight modifications for this study (Ying et al., 2011). Briefly, HCN3 WT and $\mathrm{HCN}^{-/-}$mice (P15-P30) of either sex were anesthetized and decapitated. The head was submerged in ice-cold carbogenated $\left(95 \% \mathrm{O}_{2}-5 \% \mathrm{CO}_{2}\right)$ slicing solution (containing in mM: $65 \mathrm{NaCl}, 2.5 \mathrm{KCl}, 1.25 \mathrm{NaH}_{2} \mathrm{PO}_{4}$, $26 \mathrm{NaHCO}_{3}, 0.5 \mathrm{CaCl}_{2}, 7 \mathrm{MgCl}_{2}, 105$ sucrose, 24.7 glucose, and 1.7 ascorbic acid) immediately. The brain was dissected out, the cerebellum was removed and the brain was then glued onto a platform. Coronal slices $(300 \mu \mathrm{m})$ containing the intergeniculate leaflet (IGL) were prepared on a mictrotome (Microm HM $650 \mathrm{~V}$, Thermo scientific) using ice-cold carbogenated slicing solution. The sections were cut into halves along the midline and transferred to carbogenated storage solution (containing in mM: $131 \mathrm{NaCl}, 2.5 \mathrm{KCl}, 1.25 \mathrm{NaH}_{2} \mathrm{PO}_{4}, 26 \mathrm{NaHCO}_{3}, 2 \mathrm{CaCl}_{2}, 1.2$
$\mathrm{MgCl}_{2}, 18$ glucose, 1.7 ascorbic acid, 2 sodium pyruvate and 3 myo-inositol) where they were incubated at $35^{\circ} \mathrm{C}$ for $30 \mathrm{~min}$, and kept under room temperature for at least another hour before use.

\section{Setup}

Brain slices were visualized and neurons identified using a Zeiss Axioskop 2 (Jena, Germany) equipped with a 5x objective, a $4 \mathrm{x}$ water-immersion objective and an infrared camera (VX55, Photonics, Pittsfield, USA). Ionic currents were recorded using an EPC 10 amplifier and PatchMaster software. The IGL structure and recording sites were identified using standard mouse brain atlas (Franklin and Paxinos, 2007).

\section{Ih-recordings}

Whole-cell voltage-clamp recordings were performed at $32^{\circ} \mathrm{C}$. Slices were perfused with carbogenated artificial cerebrospinal fluid (aCSF; containing in mM: $131 \mathrm{NaCl}, 2.5 \mathrm{KCl}, 1.25$ $\mathrm{NaH}_{2} \mathrm{PO}_{4}, 26 \mathrm{NaHCO}_{3}, 2 \mathrm{CaCl}_{2}, 1.2 \mathrm{MgCl}_{2}, 18$ glucose, and 1.7 ascorbic acid). $\mathrm{Ba}^{2+}$ ions $(1 \mathrm{mM})$ and Tetrodotoxin (TTX; $0.001 \mathrm{mM}$ ) were added to prevent activation of $\mathrm{K}^{+}$currents and $\mathrm{Na}^{+}$spikes. Patch pipettes $(2.5-3.5 \mathrm{M} \Omega, 1.5 \mathrm{OD} \times 1.17$ $\times 100 \mathrm{~L} \mathrm{~mm}$, Harvard apparatus, Cambridge, UK) were filled with intracellular solution (containing in mM): $140 \mathrm{KMeSO}_{4}, 10$ HEPES, $10 \mathrm{KCl}, 10$ phosphocreatine, 4 MgATP, $0.2 \mathrm{NaGTP}, 0.1$ EGTA, pH 7.3, 305 mOsm. NaGTP was freshly added daily from 100 -fold concentrated stock solutions.

\section{Electroretinography (ERG)}

ERGs were performed according to procedures described previously (Tanimoto et al., 2009). Mice were dark-adapted overnight, and then anesthetized by IP injection of ketamine $(66.7 \mathrm{mg} / \mathrm{kg})$ and xylazine $(11.7 \mathrm{mg} / \mathrm{kg})$. Body temperature was maintained at $37^{\circ} \mathrm{C}$ using a heating pad. Mouse pupils were dilated by topical administration of Mydriaticum, and custommade gold wired electrodes were placed on the corneas. ERG responses were recorded simultaneously from both eyes under dark-adapted conditions (without any background illumination). Responses were recorded using white flashes of $0.1,1,10,100$, $1,000,10,000$ and $25,000 \mathrm{mcd}^{*} \mathrm{~s} / \mathrm{m}^{2}$.

\section{Scanning-Laser Ophthalmoscopy (SLO)}

SLO imaging was performed with a HRA 1 system (Heidelberg Engineering, Heidelberg, Germany). The HRA 1 features two lasers in the short (visible) wavelength range (488 and $514 \mathrm{~nm}$ ), and in the long (infrared) wavelength range (795 and $830 \mathrm{~nm}$ ). The 488 and $795 \mathrm{~nm}$ lasers are used for fluorescein (FLA) and indocyanine green (ICG) angiography, respectively. Mice were measured according to previously described procedures (Seeliger et al., 2005). In short, mice were anesthetized by subcutaneous injection of ketamine $(66.7 \mathrm{mg} / \mathrm{kg})$ and xylazine $(11.7 \mathrm{mg} / \mathrm{kg}$ ), and their pupils dilated with tropicamide eye drops (Mydriaticum Stulln, Pharma Stulln, Stulln, Germany). A custom-made contact lens was used to avoid dehydration of the cornea. FLA was performed using a s.c. injection of $75 \mathrm{mg} / \mathrm{kg}$ body weight fluorescein-Na (University pharmacy, University of Tuebingen, Germany), and ICGA following a s.c. injection of 50 
$\mathrm{mg} / \mathrm{kg}$ body weight ICG (ICG-Pulsion, Pulsion Medical Systems AG, Munich, Germany).

\section{Circadian Experiments}

Experiments were done according to (Jud et al., 2005). Eight wild type and $10 \mathrm{HCN}^{-/-}$animals were entrained to a $12 \mathrm{~h}$ light $12 \mathrm{~h}$ dark cycle in a new cage that contained the running wheel (LD12/12) with lights switched on at 7 a.m. for 7-15 days before they were released to constant darkness (DD) or constant light (LL) for 18 days. Activity was assessed with a running-wheel and evaluated using ClockLab (Actimetrics). Activity records were double plotted in threshold format for 6-min bins. Period length was assessed by $\chi^{2}$ periodogram analysis for days $4-10$ in DD or LL. To determine light induced phase shifts, an Aschoff Type I protocol was used (Aschoff, 1977). Animals were allowed to stabilize their free-running rhythm (at least 14 days) prior to the light pulse. The circadian time (CT) at the beginning of the light pulse was calculated for every mouse individually. Fifteen minutes light pulses (400 lux, white light) were applied at CT10 (control), CT14 and CT22. Light applied at CT10 corresponds to light perceived during the subjective day and did not provoke a phase shift of behavioral activity. Light pulses at CT14 and CT22 fall at early or late subjective night and lead to phase delays or advances, respectively. Between the different light pulses, mice were allowed to stabilize their circadian oscillator for 3 weeks. Phase shifts were determined by fitting a regression line through onsets of activity before the light pulse and a second regression line through onsets of activity after the light pulse. The distance between the two regression lines on the day following the light pulse determined the amount of phase shift. Further, we tested how the two genotypes behaved in a jet-lag experiment. Animals were kept in a 12:12 LD cycle for at least 10 days. Then the LD cycle was delayed by $6 \mathrm{~h}$ (forwards or backwards) and the number of days for adaptation to the new LD cycle was measured.

\section{Behavioral Experiments}

For behavioral experiments $\mathrm{HCN}^{-/-}$animals and aged matched control wild type littermates were used. Animals were single housed at least 1 week prior to the experiments, with food and water ad-libitum under an inverse $12 \mathrm{~h}$ light/dark cycle, where lights were switched off at $7 \mathrm{a} . \mathrm{m}$. All behavioral experiments were performed during the active phase of the mice between 9 a.m. and 5 p.m. and analyzed blindly to the genotype of the animals. The (i) Morris water maze, (ii) dark-lighttransition test, and (iii) rotarod experiments were performed using the same 18 wild type and 13 knockout animals, in the given order. Furthermore, the same 10 wild type and 10 knockout animals were tested in the (i) open field and later in the (ii) fear conditioning paradigm. For all other experiments separate cohorts of animals were used as indicated.

\section{Morris Water Maze}

18 wild type and 13 knockout animals aged 10-12 weeks were tested in the morris water maze using a protocol adapted from Morris (1984) and Fritz et al. (2017). The task was performed in a circular tank (diameter: $150 \mathrm{~cm}$, height: $68 \mathrm{~cm}$, filling level:
$15 \mathrm{~cm})$ filled with water $\left(24-26^{\circ} \mathrm{C}\right)$ to $20 \mathrm{~cm}$ and surrounded by visual cues. The water was made opaque by adding sufficient amount of milk. During the training period, an escape platform $(12 \times 12 \mathrm{~cm})$ was placed in the middle of a designated target quadrant $1 \mathrm{~cm}$ below the water surface. Mice were dropped into the basin at pseudorandomized locations. On the first 3 days (training) mice were trained to find the platform by six 120 s trials. A trial ended either when an animal rested on the hidden platform for 10 or $120 \mathrm{~s}$ had elapsed (in that case, mice were guided to the platform). On day 4 the reverse hidden platform learning task was started by moving the platform to the diagonally opposite quadrant. Mice were tested in 6 runs on two consecutive days. All movement was videotaped and the tracking system Ethovision 2.2 (Noldus) was used. Parameters analyzed off-line by means of the software WinTrack were: time/distance until platform is reached, time in trained quadrant, time in other quadrants, thigmotaxis, floating, and others.

\section{Dark-Light-Transition Test}

18 wild type and 13 knockout animals were tested for anxiety in the dark-light-transition test (adapted from Crawley and Goodwin, 1980). This test was conducted in a square arena (20 $\times 45 \mathrm{~cm})$ in which a black plastic box $(20 \times 15 \mathrm{~cm})$ was used to separate adjoining dark and well-lit areas (411 lux). Mice were placed in the center of the bright area and allowed to explore the arena for $10 \mathrm{~min}$. Movements were videotaped and the tracking system used was Ethovision 2.2 (Noldus). The arena was cleaned with $70 \%$ alcohol between tests. Parameters analyzed were: latency to enter dark area, time in light area, time in dark box, number of transitions between light and dark compartment, rearing, and grooming.

\section{Rotarod}

18 wild type and 13 knockout animals were tested in the rotarod using an adaptation of a protocol previously described (Nolan et al., 2003). To measure motor coordination the accelerating rotarod apparatus (Ugo Basile) was used. During the training period, which took place on 2 days with 4 runs each day, mice were placed on the rotarod $(3 \mathrm{~cm}$ diameter) accelerating to 40 $\mathrm{rpm}$. The maximum observation time was $5 \mathrm{~min}$. During testing on the third day animals received three consecutive 2 min trials at constant speeds of $37.5,28.5,21.5$, and $11.5 \mathrm{rpm}$. The latency to fall (or pegging onto the rotating latch) was measured.

\section{Elevated Plus Maze (EPM)}

Male adult (13-20 weeks) wild type $(n=10)$ and knockout $(n$ =9) animals were tested in the EPM using a protocol described previously (Pellow and File, 1986) adapted to assess mouse behavior. The apparatus consisted of two opposite open arms, $(20 \times 5 \mathrm{~cm})$ and two opposite closed arms $(20 \times 5 \times 14 \mathrm{~cm})$ connected to a central platform $(5 \times 5 \mathrm{~cm})$ to form a cross. The maze was elevated $50 \mathrm{~cm}$ from the floor. Illumination was adjusted to 50 lux. The animals were placed at the center of the maze with the nose in the direction of one of the closed arms, and observed for $5 \mathrm{~min}$, according to the following parameters: number of entries in the open and closed arms, and time of permanence in each of them (i.e., the time spent by the animal in 
the open and closed arms). An entry was defined as all four paws having crossed the imaginary line between an arm and the central area. On removal of each mouse, the maze floor was carefully wiped with $70 \% \mathrm{EtOH}$. The behavior of all animals was recorded using a video camera and analyzed offline using the TSE maze systems software.

\section{Marble-Burying Test}

Based on the protocol previously published by Deacon (2006), 10 wild type and 10 knockout animals (17-19 weeks old) were tested in their home cage $(35.5 \times 20.7 \mathrm{~cm})$ filled with fresh bedding to $5 \mathrm{~cm} .12$ glass marbles $(1.4 \mathrm{~cm}$ diameter) were arranged in 3 $\times 4$ equidistant rows. After $30 \mathrm{~min}$ under low-light conditions, the number of marbles that were at least covered for $2 / 3$ was determined.

\section{Forced Swimming Test (FST)}

Using an adaptation of the FST protocol described in Castagné et al. (2011), 20 wild type and 20 knockout animals were tested in standard 51 glass beakers (height $25 \mathrm{~cm}$; Schott) filled with water $\left(26 \pm 0.3^{\circ} \mathrm{C}\right)$ to $10 \mathrm{~cm}$, so that the animals could neither touch the bottom with their tails, nor could escape from the top. Two beakers were separated by an opaque screen, so that two animals (WT and litter-matched $\mathrm{HCN}^{-/-}$) could be tested at the same time. Between sessions, the beakers were cleaned thoroughly and fresh water was added. At the start of each test, an animal was gently picked up by its tail from the home cage and rapidly placed into the middle of the beaker. At the time the animal was placed in the water, the recording time was started and the duration of each standard FST was set to $6 \mathrm{~min}$. After the test, the animal was removed from the water, dried with a towel and put into a warm cage (temperature of bedding $31-33^{\circ} \mathrm{C}$ ) for $15 \mathrm{~min}$ before returning to their home cage. The entire session was videotaped (Logitech Quick Cam) and analyzed off-line using a software (Winrat.exe version2.3.1) by an experienced experimenter. Mice were tested again $24 \mathrm{~h}$ later.

\section{Open Field Test}

To assess locomotor activity, wild type $(n=10)$ and $\mathrm{HCN}^{-/-}$ $(n=10)$ animals at an age of 6-9 weeks were tested in the open field under low light conditions, using a protocol adapted from Leitinger et al. (1994). The open field chamber used was a TSE conditioning chamber $(25 \times 25 \times 25 \mathrm{~cm})$ covered with acoustic foam (Conrad electronics). The test was initiated by placing mice in the middle of the open field and allowing them to move freely for $10 \mathrm{~min}$, while being tracked by light barriers. The chamber was cleaned with $70 \% \mathrm{EtOH}$ between tests. Testing was repeated $24 \mathrm{~h}$ later. Parameters analyzed were distance moved, velocity, and time being active (movement speed $>3 \mathrm{~cm} / \mathrm{s}$ ).

\section{Fear Conditioning}

Male wild type $(n=10)$ and $\mathrm{HCN}^{-/-}(n=10)$ animals at an age of 6-9 weeks were used. The setup for the fear conditioning test has been described and displayed in detail before (Kamprath and Wotjak, 2004). Briefly, the experiments were performed in two contexts: (1) the shock chamber was a cubic-shaped box with a metal grid for shock application (conditioning chamber,
TSE) and (2) the neutral test context, which was cylindrically shaped $(25 \mathrm{~cm}$ diameter) and made of transparent acrylic glass, with wood shavings as bedding. The two contexts were cleaned thoroughly after each trial with differently smelling detergents, and bedding was changed. For conditioning (d0), mice were placed into the shock chamber. After $180 \mathrm{~s}$ a tone was presented $(9 \mathrm{kHz}, 80 \mathrm{~dB}, 30 \mathrm{~s})$, which co-terminated with a single scrambled electric foot shock ( $2 \mathrm{~s}, 0.7 \mathrm{~mA})$. Animals remained in the shock chamber for another $30 \mathrm{~s}$ before they were returned to their home cages. On day 1 and 2 after conditioning, mice were exposed either to the tone (180s) in the neutral test context (d1) or to the conditioning context (180s). One week later the exposure to the tone in the neutral or the conditioning context was repeated. The behavioral performance was videotaped by a video camera (Eneo VK-1316s, TSE), and animals' behavior was analyzed off-line by a trained observer who was unaware of the genotypes. Freezing behavior was defined as immobility except for respiration movements and served as a measure of fear memory.

\section{Statistical Analysis}

Data were analyzed in GraphPad Prism (version 7.01) using $\chi^{2}$, unpaired $t$-test or two-factor analysis of variance for repeated measures and the post-hoc Bonferroni test for multiple comparisons if appropriate. Statistical significance was accepted if $P \leq 0.05$.

\section{RESULTS}

For the present study we used the previously published knockout mouse line, $\mathrm{HCN}^{-/-}$, in which $\mathrm{HCN} 3$ is globally deleted (Fenske et al., 2011). HCN3-deficient mice were born at the expected Mendelian ratio, were fertile, and showed no immediately visible physical abnormalities. The body weights were similar in wild type and $\mathrm{HCN}^{-/-}$mice (wild type: 29.64 $\left.\pm 1.29 \mathrm{~g} ; \mathrm{HCN3}^{-/-}: 31.27 \pm 1.31 \mathrm{~g} ; t=0.8866, p>0.05\right)$ 12 weeks after birth. Furthermore, organ specific laboratory parameters including plasma cholesterol, lipoprotein panel, liver function tests (total plasma proteins, transaminases alkaline phosphatase), exocrine pancreatic function (amylase and lipase) and blood glucose levels were indistinguishable between wild type and $\mathrm{HCN}^{-/-}$animals under baseline conditions (Table 1). Moreover, plasma electrolyte concentrations were similar in both group of mice (see Table 1) with only mildly decreased $\mathrm{Ca}^{2+}$ concentrations and urea levels in $\mathrm{HCN}^{-/-}$mice as compared to wild type mice. Creatinine and uric acid were normal.

In order to detect $\mathrm{HCN} 3$ protein we previously generated a HCN3 antibody raised against the C-terminus of $\mathrm{HCN} 3$ as described in Mistrik et al. (2005). In the absence of preabsorption against $\mathrm{HCN}^{-/-}$lysates unspecific signal including nuclear staining was present (not shown). Nuclear staining of HCN3 using non preabsorbed antibody seems to be present in Ying et al. (2011) and Peng et al. (2017). To reduce unspecific binding we therefore only used HCN3 antibody preabsorbed against $\mathrm{HCN}^{-/-}$brain lysates throughout this study as described in Fenske et al. (2011). We first confirmed the specificity of the HCN3 antibody (Supplementary Figure 1) in the brain. A side 
TABLE 1 | Blood plasma parameters.

\begin{tabular}{|c|c|c|c|c|}
\hline Parameter & WT & $\mathrm{HCN}^{-/-}$ & Unit & Method \\
\hline $\mathrm{Na}^{+}$ & $158 \pm 0.51$ & $156.5 \pm 1.47$ & $\mathrm{mmol} / \mathrm{l}$ & Ion selective electrode \\
\hline $\mathrm{K}^{+}$ & $3.86 \pm 0.12$ & $3.73 \pm 0.08$ & $\mathrm{mmol} / \mathrm{l}$ & Ion selective electrode \\
\hline $\mathrm{Ca}^{2+}$ & $2.07 \pm 0.03$ & $1.96 \pm 0.03^{\star}$ & $\mathrm{mmol} / \mathrm{l}$ & Photometric Color Test \\
\hline $\mathrm{Cl}^{-}$ & $114.58 \pm 0.62$ & $114.25 \pm 1.10$ & $\mathrm{mmol} / \mathrm{l}$ & Ion selective electrode \\
\hline $\mathrm{PO}_{4}^{3-}$ & $1.71 \pm 0.07$ & $1.73 \pm 0.08$ & $\mathrm{mmol} / \mathrm{l}$ & Photometric UV Test \\
\hline Creatinine & $0.32 \pm 0.01$ & $0.32 \pm 0.0036$ & $\mathrm{mg} / \mathrm{dl}$ & Kinetic Color Test \\
\hline Urea & $46.62 \pm 1.78$ & $38.95 \pm 1.67^{\star \star}$ & $\mathrm{mg} / \mathrm{dl}$ & Kinetic UV Test \\
\hline Uric acid & $0.47 \pm 0.18$ & $0.27 \pm 0.16$ & $\mathrm{mg} / \mathrm{dl}$ & Enzymatic Color Test \\
\hline \multicolumn{5}{|l|}{ LIPID METABOLISM } \\
\hline Cholesterol & $75.92 \pm 4.04$ & $76.60 \pm 4.69$ & $\mathrm{mg} / \mathrm{dl}$ & Enzymatic Color Test \\
\hline Triglycerides & $117.26 \pm 12.12$ & $111.59 \pm 9.13$ & $\mathrm{mg} / \mathrm{dl}$ & Enzymatic Color Test \\
\hline LDL & $15.93 \pm 0.86$ & $16.45 \pm 1.14$ & $\mathrm{mg} / \mathrm{dl}$ & Enzymatic Color Test \\
\hline HDL & $50.67 \pm 2.84$ & $49.93 \pm 2.98$ & $\mathrm{mg} / \mathrm{dl}$ & Enzymatic Color Test \\
\hline \multicolumn{5}{|l|}{ LIVER FUNCTION } \\
\hline Total protein & $5.13 \pm 0.1$ & $4.85 \pm 0.12$ & $\mathrm{~g} / \mathrm{dl}$ & Photometric Color Test \\
\hline Alanine transaminase & $22.35 \pm 2.04$ & $21.38 \pm 2.52$ & $\mathrm{U} / \mathrm{I}$ & Kinetic UV Test \\
\hline Aspartate transaminase & $62.47 \pm 6.93$ & $54.63 \pm 5.94$ & $U / I$ & Kinetic UV Test \\
\hline Alkaline phosphatase & $126.94 \pm 8.82$ & $125.25 \pm 13.40$ & $U / I$ & Kinetic Color Test \\
\hline Ferritin & $47.29 \pm 4.62$ & $45.23 \pm 4.74$ & $\mu g / l$ & Immuno-turbidimetric Test \\
\hline Transferrin & $147.41 \pm 2.27$ & $145.23 \pm 1.55$ & $\mathrm{mg} / \mathrm{dl}$ & Immuno-Turbidimetric Test \\
\hline \multicolumn{5}{|c|}{ PANCREATIC FUNCTION } \\
\hline Glucose & $155.35 \pm 6.41$ & $162.21 \pm 6.48$ & $\mathrm{mg} / \mathrm{dl}$ & Enzymatic UV Test \\
\hline Alpha-amylase & $2310.00 \pm 72.97$ & $2393.25 \pm 58.52$ & $U / I$ & Kinetic Color Test \\
\hline Lipase & $53.77 \pm 2.45$ & $51.77 \pm 2.07$ & $U / I$ & Kinetic Color Test \\
\hline Creatine kinase & $578.12 \pm 42.69$ & $497.38 \pm 46.55$ & $U / I$ & Kinetic UV Test \\
\hline
\end{tabular}

Parameters were assessed from blood plasma of 10 wild type and $11 \mathrm{HCN}^{-1-}$ animals. ${ }^{*} p<0.05,{ }^{* *} p<0.01$.

by side comparison of cryosections prepared from wild type and $\mathrm{HCN}^{-/}$brains revealed the presence of $\mathrm{HCN} 3$ in wild type and the absence of $\mathrm{HCN} 3$ in the $\mathrm{HCN}^{-/-}$brain, indicating that the $\mathrm{HCN} 3$ antibody specifically detects $\mathrm{HCN} 3$ protein. There is however unspecific staining in vascular structures including the choreoid plexus. Immunohistochemical (IHC) experiments and Western blots (For examples see Supplementary Figures 1, 2, respectively) revealed that $\mathrm{HCN} 3$ is broadly expressed in the CNS, in particular in the cortex, amygdala, hippocampus, the hypothalamus, pituitary gland, thalamus, brainstem, olfactory system, retina, and the intergeniculate leaflet (IGL) of the circadian system (Table 2). Inspired by the distinct expression pattern of $\mathrm{HCN} 3$ in the CNS we characterized the expression and function of these channels in the circadian system and their role for behavior in more detail.

\section{Role of HCN3 Channels in the Circadian System}

Circadian rhythms, including timing of wakefulness and sleep, are mainly controlled by the suprachiasmatic nucleus of the hypothalamus (SCN) which acts as a master clock controlling circadian behavior (Figure 1; Albrecht et al., 2012). This clock is synchronized (or entrained) to day-night rhythm by the activity of brain regions, which provide information about light (photic entrainment by the retina), arousal or availability of food (both non-photic entrainment). Among these regions, the intergeniculate leaflet (IGL) has been suggested to be critical for the integration and for transmission of these photic and non-photic entrainment cues to the SCN (Figure 1; Delogu et al., 2012). The IGL is located upstream of the SCN within the thalamus forming a leaflet in between the medial and lateral geniculate nuclei. The dominant entrainment cue for the circadian system is light (photic entrainment). Light input signal for the circadian system is detected by the retina and transferred via the retinohypothalamic tract (RHT) to the SCN and the IGL. Given the central role of the retina, the SCN, and the IGL for the control of circadian rhythm we next analyzed the expression and the functional role of HCN3 channels in these regions.

IHC experiments revealed distinct immune labeling for $\mathrm{HCN} 3$ in the retina (Table 2, Figure 2A). HCN3 signal is mainly found in the inner and to a lesser extent in the outer plexiform layer, which harbor the retinal synapses between bipolar and ganglion cells, and between photoreceptors and bipolar cells, respectively. Retinal in vivo imaging and fluorescein angiography via scanning-laser ophthalmoscopy (SLO) did not provide any evidence for morphological changes to neuroretinal or vascular structures in $\mathrm{HCN}^{-/}-$animals (Figure 2B). Retinal function, analyzed via electroretinography (ERG) under scotopic (darkadapted) and photopic (light-adapted) conditions, was normal 
TABLE 2 | Expression of HCN3 channels.

\begin{tabular}{|c|c|}
\hline Name of the structure & Expression level \\
\hline \multicolumn{2}{|l|}{ Hypothalamus ${ }^{\mathrm{a}}$} \\
\hline Hypophysis & ++ \\
\hline Nucleus dorsomedialis & ++ \\
\hline Nucleus hypothalamicus anterior & ++ \\
\hline Nucleus lateralis & ++ \\
\hline Nucleus paraventricularis & ++ \\
\hline Nucleus preopticus medialis & ++ \\
\hline Nucleus supraopticus & ++ \\
\hline Suprachiasmatic nucleus & - \\
\hline \multicolumn{2}{|l|}{ Thalamus } \\
\hline Corpus geniculatum mediale & - \\
\hline Intergeniculate leaflet & ++ \\
\hline Lateral geniculate nucleus dorsal part & - \\
\hline Lateral geniculate nucleus ventral part & ++ \\
\hline Nucleus habenularis laterlais & ++ \\
\hline Nucleus habenularis medialis & ++ \\
\hline Nucleus laterodorsalis & ++ \\
\hline Nucleus paraventricularis Thalamicus & ++ \\
\hline Nucleus reticularis & ++ \\
\hline \multicolumn{2}{|l|}{ Brainstem } \\
\hline Locus coeruleus & ++ \\
\hline Nucleus parabrachialis lateralis & ++ \\
\hline Nucleus parabrachialis medialis & ++ \\
\hline Nucleus subparabrachialis & ++ \\
\hline Nucleus tegmentalis, laterodorsal part & ++ \\
\hline Periaqueductal gray & ++ \\
\hline Raphe nuclei dorsal part & ++ \\
\hline Raphe nuclei medial part & ++ \\
\hline Substantia nigra pars compacta & ++ \\
\hline \multicolumn{2}{|l|}{ Limbic system } \\
\hline Basolateral Amygdala & ++ \\
\hline Hippocampus, str. Lacunosum moleculare & + \\
\hline \multicolumn{2}{|l|}{ Main olfactory bullba } \\
\hline Glomerular layer & ++ \\
\hline Internal plexiform layer & ++ \\
\hline \multicolumn{2}{|l|}{ Telencephalon } \\
\hline Cortex ${ }^{a}$ & + \\
\hline Nucleus basalis & ++ \\
\hline Striatum & ++ \\
\hline Cerebellum ${ }^{\mathrm{a}}$ & + \\
\hline \multicolumn{2}{|l|}{ Retina $^{a}$} \\
\hline Inner plexiform layer & ++ \\
\hline Outer plexiform layer & + \\
\hline \multicolumn{2}{|l|}{ Outside CNS } \\
\hline Heart & + \\
\hline
\end{tabular}

Expression data was obtained using Immunohistochemistry. ${ }^{\text {a Presence of HCN3 } \text { protein }}$ was confirmed with Western blots.

in $\mathrm{HCN}^{-/-}$mice (Figure 2C). In particular, amplitude and shape of the b-wave, which reflects directly the activity of bipolar cells in the inner retina and indirectly activity of photoreceptors and successful synaptic transmission at the first synapse (Tanimoto et al., 2009), were normal (Figure 2C, left). Overall, results obtained from ERG studies indicate no obvious role of HCN3 channels for processing of electrical signals in the retina.

Given the central role of the $\mathrm{SCN}$ for the regulation of circadian rhythm, we next performed IHC experiments in this specific nucleus and in neighboring hypothalamic nuclei. Interestingly, the immunosignal for $\mathrm{HCN} 3$ in the $\mathrm{SCN}$ was very weak compared to the surrounding cell populations (Supplementary Figure 3). HCN3 is abundantly expressed in other nuclei of the hypothalamus (Nucl. paraventricularis, Nucl. dorsomedialis, Nucl. lateralis, Nucl. hypothalamicus anterior and Nucl. preopticus medialis; Table 2; Supplementary Figure 4), which are not related to circadian function. However, we did not find any evidence for specific functional defects of $\mathrm{HCN}^{-/-}$mice including corticosterone, fluid and electrolyte homeostasis controlled by these nuclei (Supplementary Figure 4). Remarkably, and in line with previous reports (Ying et al., 2011), we were able to confirm pronounced expression of $\mathrm{HCN} 3$ in the IGL (Figures 3A,B; Table 2), which was absent in $\mathrm{HCN}^{-/-}$ animals (Supplementary Figure 1). In addition to HCN3, HCN2 channels are expressed in the IGL, while HCN1 and HCN4 are hardly detectable. $\mathrm{I}_{\mathrm{h}}$ recordings in wild type and $\mathrm{HCN}^{-/-}$ mice confirm that HCN3 channels contribute to $I_{h}$ in the IGL (Figure 3B). Furthermore, in WT 28/108 neurons in the IGL $(25 \%)$ displayed $\mathrm{I}_{\mathrm{h}}$ while in $\mathrm{KO}$ significantly less cells, $18 / 151$ $(10 \%)$, displayed this current $\left(\chi^{2}=8.456 ; p<0.01\right)$ (Figure 3C). This indicates that different populations of neurons are present in the IGL, one population in which $\mathrm{I}_{\mathrm{h}}$ is entirely conferred by $\mathrm{HCN} 3$ and a second population which express a $\mathrm{HCN}$ isoform that is not affected by the knockout of $\mathrm{HCN} 3$, most likely HCN2.

To evaluate whether HCN3 channels in the IGL play a substantial role in the regulation of the circadian clock, we characterized the daily activity in wild type and $\mathrm{HCN}^{-/-}$mice (Figure 4). Animals were entrained to a $12 \mathrm{~h}$ light/dark (LD) cycle $(12: 12 \mathrm{LD})$ for 15 days and then released into constant darkness (DD) for 18 days (Figure 4A). The two groups displayed similar free-running activity rhythms (period length) under DD (WT: $23.49 \pm 0.03 \mathrm{~h}$; KO: $23.6 \pm 0.07 \mathrm{~h} ; t=1.307, p>0.05$ ) as well as under LL ( $24 \mathrm{~h}$ light; WT: $25.14 \pm 0.12 \mathrm{~h}$; KO: $25.45 \pm 0.13 \mathrm{~h} ; t=1.705, p>0.05)$ conditions (Figure 4B). Interestingly, the precision of activity onset was similar in the two genotypes under LD (Figure 4A) as well as under DD (Figure 4D) conditions. Furthermore, the two groups did not differ significantly in total activity under LD (WT: 24,091 \pm $1,960 \mathrm{rev}$ KO: $20,439 \pm 2,357 \mathrm{rev} ; t=1.152, p>0.05)$ and LL (WT: 7,200 $\pm 1,241 \mathrm{rev}$ KO: $8,118 \pm 1613 \mathrm{rev} ; t=0.4325, p$ $>0.05$ ) conditions (Figure 4C), however under DD conditions the $\mathrm{HCN}^{-/-}$animals displayed significantly less activity (17925 \pm 1945 wheel-revolutions, $n=10$ ) compared to their controls (23482 \pm 1555 wheel-revolutions, $n=8 ; t=2.148, p<0.05$ ). These results suggest that $\mathrm{HCN} 3$ channels can affect the total amount of activity in the absence of a light cue, but do not play a major role in the regulation of circadian timing under constant lighting conditions.

Because it is well-known that cAMP-dependent signaling and neuronal excitation are both involved in the light-dependent entrainment process of the circadian clock, we tested the 

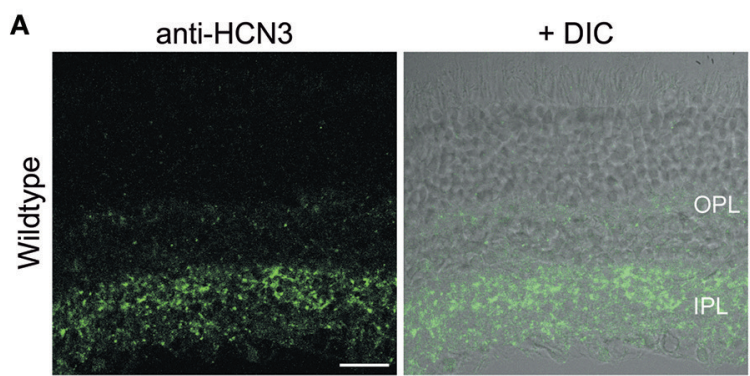

B

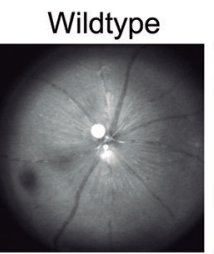

HCN3 -/-
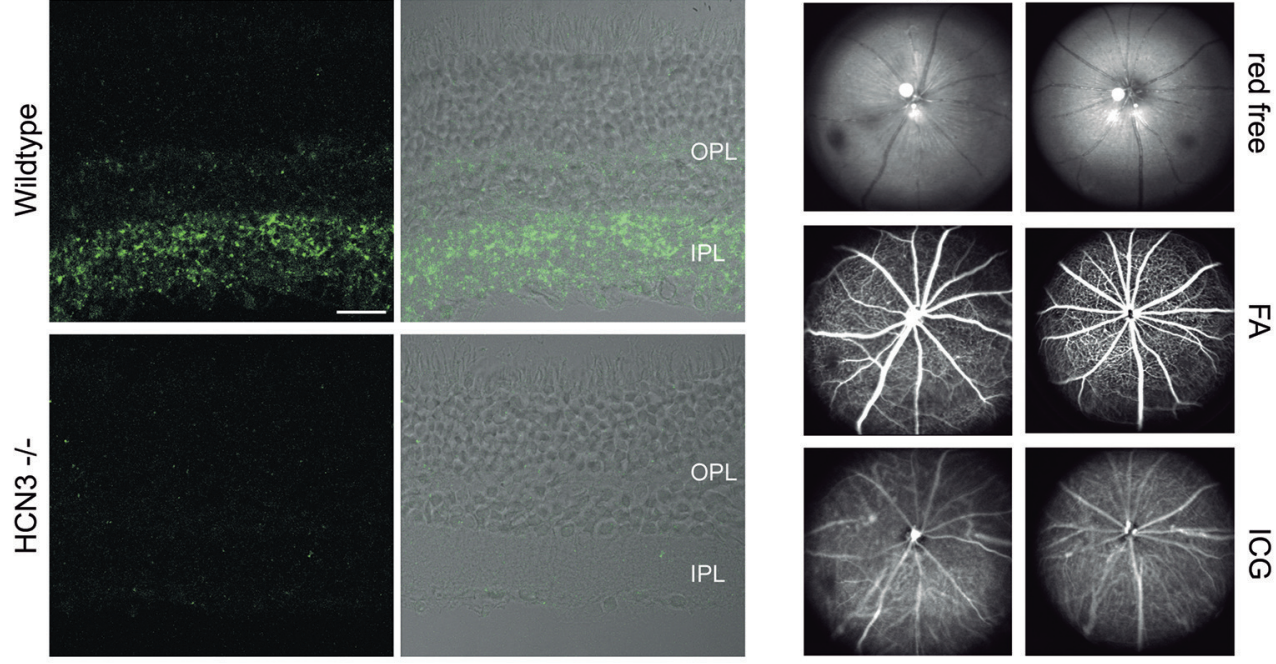

C
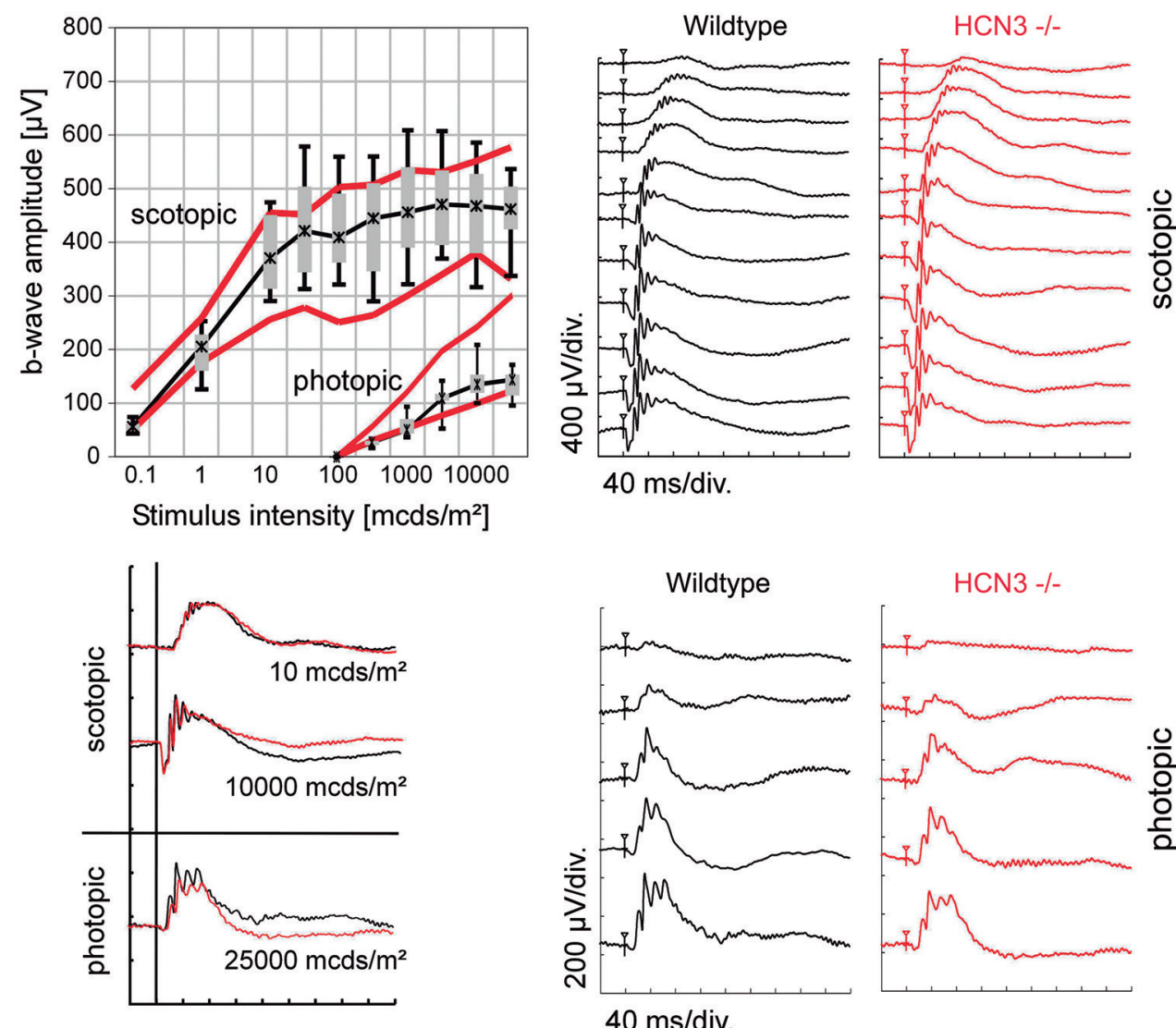

HCN3 -/-

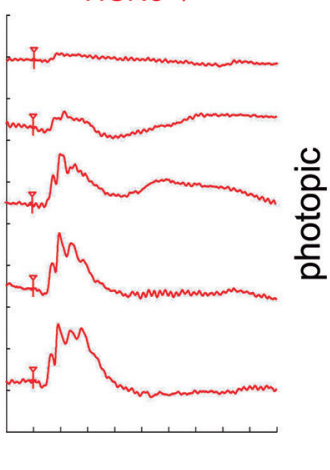

FIGURE 2 | Characterization of retinal structure and function in $\mathrm{HCN}^{-/-}$mice. (A) Expression of HCN3 in the retina. Wild type mice express HCN3 (green) in the inner plexiform layer (IPL) and the outer plexiform layer (OPL). The immunolabeling is absent in $\mathrm{HCN}^{-/}-$mice. For visualization of the retinal layers, confocal fluorescent images are overlaid with DIC images of the same area. Scale bar, $20 \mu \mathrm{m}$. (B,C) Examination of retinal structure and function in vivo [wild type control mice $(n=5)$ and $\mathrm{HCN}^{-/-}(n=5)$, age 3 months]. (B) Retinal imaging and angiography. Mice were examined with native SLO (red-free, $\left.514 \mathrm{~nm}\right)$, fluorescein angiography $(488 \mathrm{~nm}$, barrier filter at $500 \mathrm{~nm})$, and indocyanine green (ICG) angiography $(795 \mathrm{~nm}$, barrier filter at $800 \mathrm{~nm})$. HCN3 ${ }^{-/-}$mice show no signs of neuroretinal or vascular alterations. (C) Retinal function assessment via ERG. Top right, Representative scotopic ERG intensity series (dark-adapted conditions). Bottom right, Representative photopic ERG intensity series (light-adapted conditions). Top left, Quantitative scotopic and photopic b-wave amplitude data from $\mathrm{HCN}^{-1-}$ mice (box plot). Boxes: 25-75\% quartile range, whiskers: 5 and $95 \%$ quantiles, asterisks: median. As a control, red bolded lines indicate the 25 and $75 \%$ quartile of the wild type group. bottom left, Overlay of representative ERG recordings under scotopic $\left(0.01\right.$ and $\left.10 \mathrm{~cd} \mathrm{~d}^{*} \mathrm{~s} / \mathrm{m}^{2}\right)$ and photopic $\left(25 \mathrm{~cd} \mathrm{~d}^{*} / \mathrm{m}^{2}\right)$ conditions. Wild type black traces, HCN3-/red traces. 
A
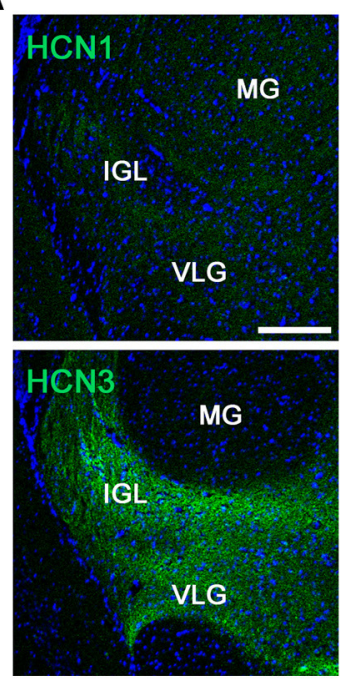
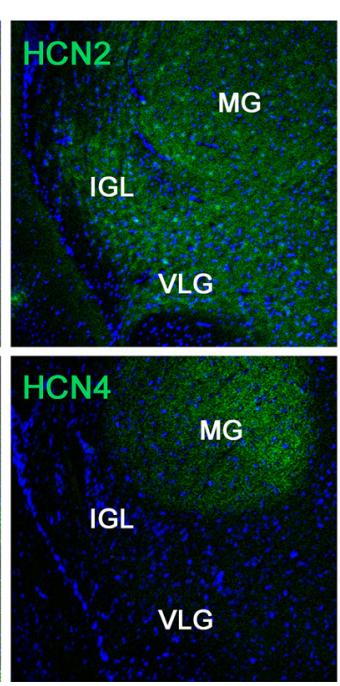

B

WT
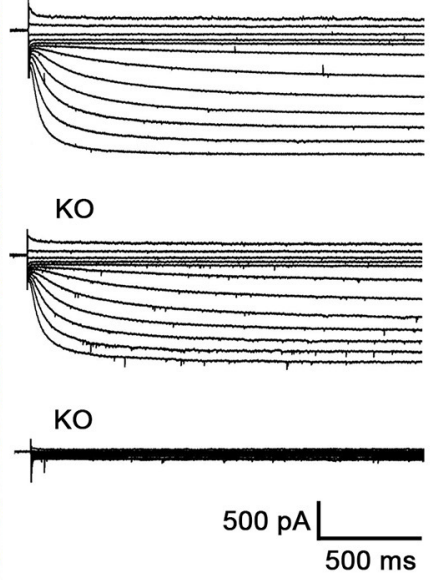

C

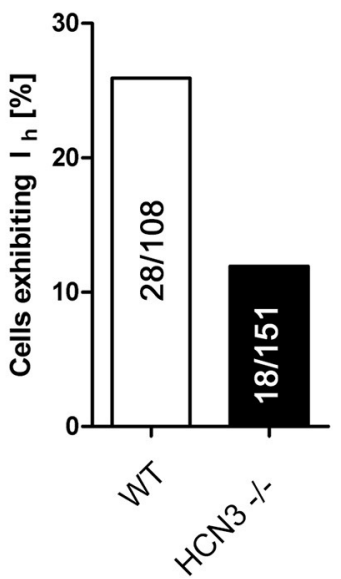

FIGURE 3 | HCN3 channels are expressed in the intergeniculate leaflet and contribute to $\mathrm{h}$. (A) Expression of HCN channel subtypes in the intergeniculate leaflet (IGL), medial geniculate nucleus (MG) and ventral geniculate nucleus (VLG); Scale bar, $200 \mu \mathrm{m}$. (B) Family of current traces for lh recordings in acute slice preparations containing the IGL. Upper panel: recordings from WT preparations. In slices prepared from $\mathrm{HCN}^{-/-}$mice, two populations of cells were present, one containing lh (middle panel), which is most likely carried by HCN2, and one without $\mathrm{I}_{\mathrm{h}}$ (lower panel). (C) Number of tested IGL neurons exhibiting the $\mathrm{I}_{\mathrm{h}}$ current in relation to the number of tested cells given in \%.

potential role of $\mathrm{HCN} 3$ channels in the regulation of the response of the circadian clock to a nocturnal light stimulus. We analyzed the clock resetting behavior of $\mathrm{HCN}^{-/-}$mice and compared it to wild type animals. Mice of both genotypes were held in DD for at least 14 days and then exposed to a 20-min light pulse. Subsequently, the animals were kept for an additional 16 days in DD, and their phase-shifts were assessed. As expected, both genotypes, control and $\mathrm{HCN}^{-/-}$mice, did not display phase-shifts when subjected to light at CT10 (data not shown), because this time point corresponds to subjective day (CT0-12), where the clock is insensitive to light. Exposure to a light pulse in the early subjective night (CT14), evoked normal phase delays in both control and $\mathrm{HCN}^{-/-}$mice $(-93.29 \pm 12.88 \mathrm{~min},-107.8$ $\pm 14.24 \mathrm{~min}$, respectively; $t=0.7181, p>0.05$; Figure 4D, top panels). Likewise, application of a light pulse in the late subjective night (CT22) evoked a phase advance in both genotypes (WT: 39 \pm 7.01 ; KO: $28.44 \pm 4.45 \mathrm{~min} ; t=1.336, p>0.05$; Figure 4D bottom panels). There was no difference in the amount of phase delays and phase advances between $\mathrm{HCN}^{-/-}$mice and wild type control animals (Figure 4D). Overall, these results suggest that the HCN3 channel does not play a major role in the light-induced resetting mechanism of the circadian clock.

In a next step, we tested how the two genotypes behaved in a jet-lag experiment. Animals were kept in a 12:12 LD cycle for at least 10 days. Then the LD cycle was delayed by $6 \mathrm{~h}$ and the number of days for adaptation to the new LD cycle was measured. For both genotypes this backward shift of the clock was similar and took $<2$ days (WT: $1.63 \pm 0.26$ days; KO: $1.1 \pm 0.1$ days; $t=2.065, p>0.05$; Figure 4E). Advancing the LD cycle by $6 \mathrm{~h}$ took the animals of both genotypes around 4 days (WT: $3.88 \pm$ 0.44 days; KO: $4.1 \pm 0.67$ days; $t=0.2592$, $p>0.05$; Figure $4 \mathrm{E}$ ). These observations indicate that the HCN3 channel does not play an important role in the speed of adaptation in the behavior of mice to a new LD cycle.

\section{Role of HCN3 Channels for the Control of Behavior}

We performed a set of behavioral tasks for motor learning, spatial learning, and memory, exploratory behavior as well as emotionality such as depression-related behavior and stereotypic perseverative behavior.

To evaluate whether HCN3 channels are involved in the control of motor function, wild type and $\mathrm{HCN}^{-/-}$mice were trained for 2 days with 4 trials per day to balance on a rod accelerating from 4 to $40 \mathrm{rpm}$ over $5 \mathrm{~min}$, and the latency at which mice fell from the accelerating rod was recorded (Figures 5A,B). Using this rotarod protocol, motor coordination, skilled movements and motor learning can be evaluated. Both, WT and $\mathrm{HCN} 3^{-/}$mice showed motor learning on the rotarod (accelerating mode) as indicated by the increase in fall-off latencies [Trial: $F_{(7,203)}=40.22, p<0.0001 ; 2$-way ANOVA for repeated measures] with no genotype differences [GT: $F_{(1,29)}$ $=0.3251, p>0.05 ; \mathrm{GT} \times$ Trial: $F_{(7,203)}=0.5523, p>0.05 ;$ Figure 5B]. On day 3, mice were placed on a rotarod of constant speed (fixed mode) at $37.5,28.5,21.5$, and $11.3 \mathrm{rpm}$, and the latencies at which mice fell from the rod were determined. In the fixed mode, fall-down latencies were clearly dependent on the speed of the rod $\left[\mathrm{rpm}: F_{(3,87)}=70.73, p<0.0001 ; 2\right.$-way ANOVA for repeated measures] with no genotype differences [GT: $F_{(1,29)}=0.054, p>0.05 ; \mathrm{GT} \times \mathrm{rpm:} F_{(3,87)}=0.042$, $p>0.05$; Figure 5C]. These findings indicate that $\mathrm{HCN} 3$ is dispensable for motor learning and motor coordination.

Exploration-based behavior was tested using the open field test (Figure 5D). For this test, mice were exposed to an Open 


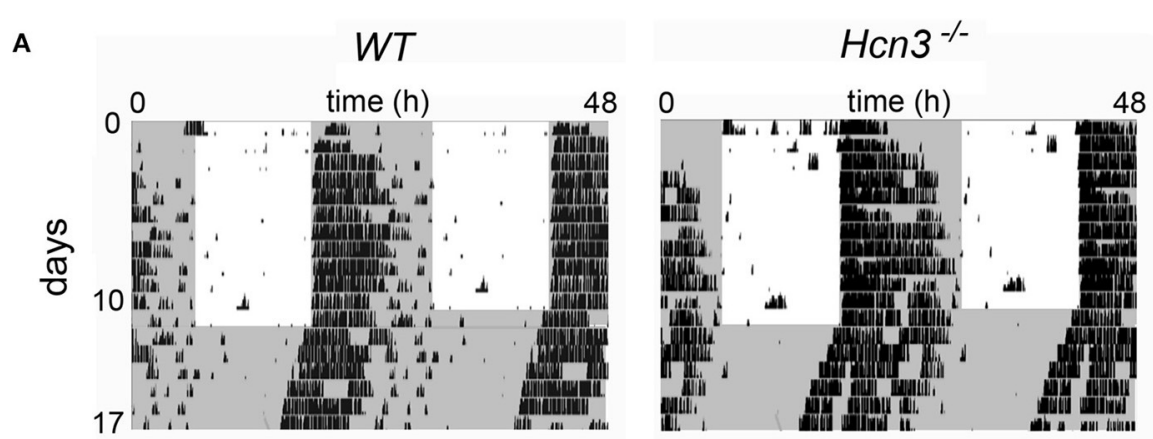

B

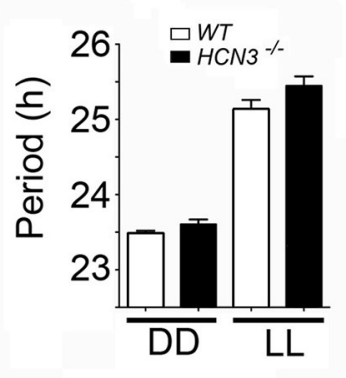

C

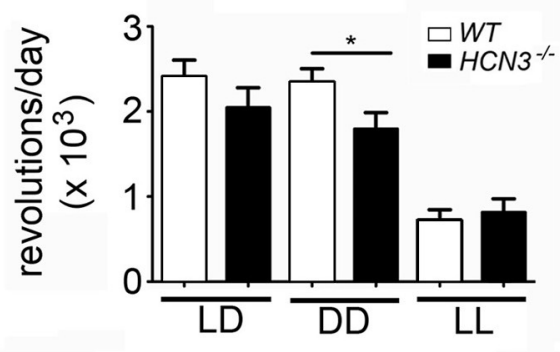

$\mathrm{HCn}^{-/-}$

D
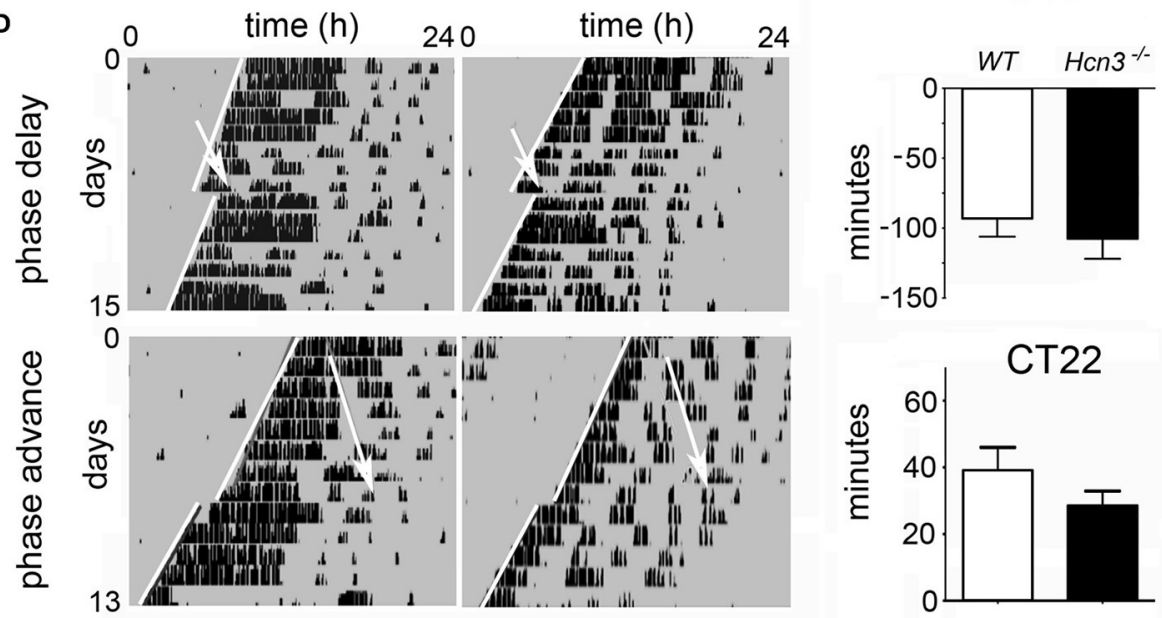

E

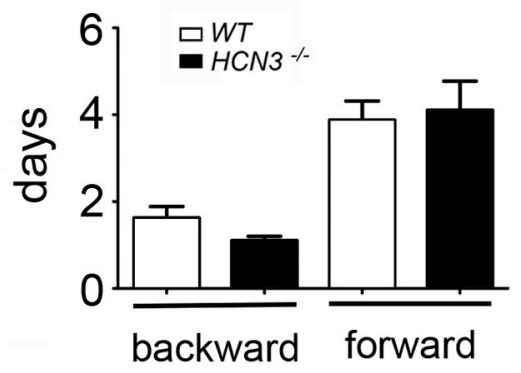

FIGURE 4 | Continued 
FIGURE 4 | Circadian clock parameters of HCN3 ${ }^{-/-}$mice. (A) Wheel-running activity of wild type (left) and HCN3 ${ }^{-/-}$(right) mice. Gray areas indicate darkness and white areas light. The actograms are double plotted and display two consecutive days per line. (B) Quantification of the period under constant darkness (DD) and constant light (LL) of wild type (black bars) and HCN3 ${ }^{-/-}$(white bars) mice. Under DD conditions the periods were $23.49 \pm 0.03 \mathrm{~h}(n=7)$ and $23.6 \pm 0.07 \mathrm{~h}(n=9)$ and under $\mathrm{LL}$ conditions they were $25.14 \pm 0.12 \mathrm{~h}(n=7)$ and $25.45 \pm 0.13 \mathrm{~h}(n=9)$, respectively. (C) Total activity in the two genotypes under light dark (LD), DD, and LL conditions. Wheel revolutions (rev) per day were counted and were as follows: LD conditions, WT $24091 \pm 1960$ rev $(n=8)$, HCN3 $-/-20439 \pm 2357$ rev $(n$ $=10)$, DD conditions, WT $23482 \pm 1555$ rev $(n=8), \mathrm{HCN}^{-/}-17925 \pm 1945 \mathrm{rev}(n=10), \mathrm{LL}$ conditions, WT $7200 \pm 1241 \mathrm{rev}(n=8)$, HCN3 $-/-8118 \pm 1613 \mathrm{rev}$ $(n=10)$. (D) Clock-resetting in response to a light pulse at CT14 (phase delay) and CT22 (phase advance). White arrows indicate the time of application of the light pulse. White lines delineate activity onset, before (top) and after (bottom) the light pulse. The phase delays were: WT $-93.29 \pm 12.88$ min $(n=7)$ and HCN3 $-/-$ $-107.8 \pm 14.24 \mathrm{~min}(n=10)$. The phase advances were: WT $39 \pm 7.01 \mathrm{~min}(n=5)$ and HCN3 $-/-28.44 \pm 4.45 \mathrm{~min}(n=9)$. (E) Adaptation time to a jet-lag delaying the onset of light by $6 \mathrm{~h}$ (backward shift) or advancing the onset of light by $6 \mathrm{~h}$ (forward shift). The adaptation times were: backward, WT $1.63 \pm 0.26$ days $(n=8)$ and $\mathrm{HCN}^{-/}-1.1 \pm 0.1$ days $(n=10)$, forward, WT $3.88 \pm 0.44$ days $(n=8)$ and $\mathrm{HCN}^{-/-} 4.1 \pm 0.67$ days $(n=10)$. All values are Mean \pm SEM, unpaired two-tailed t-test, ${ }^{*} p<0.05$.

Field for $10 \mathrm{~min}$ on two consecutive days. On the second day of testing, $\mathrm{KO}$ animals showed reduced horizontal exploration [GT: $F_{(1,18)}=11.97, p<0.01 ; 2$-way ANOVA for repeated measures; Figure 5E], which declined to the same extent in both genotypes [Day: $F_{(1,18)}=26.0, p<0.0001$; GT $\times$ Day: $\left.F_{(1,18)}=0.0666, p>0.05\right]$. The main effect of genotype can be ascribed to a general increase in immobility in KO mice [GT: $F_{(1,18)}=8.034, p<0.05$; Figure 5F], which was also observed in circadian experiments under constant darkness. In contrast, vertical exploration [rearing; GT: $F_{(1,18)}=0.0023, p>0.05$; Figure 5G] and grooming [GT: $F_{(1,18)}=0.0044, p>0.05$; Figure $\mathbf{5 H}$ ] were unaffected.

In the elevated plus maze (Figure 5I), another independent test for exploration-based behavior, both, WT and $\mathrm{HCN}^{-/-}$ mice avoided exploration of the aversive open arms to the same extent (Figures 5J,K) and showed comparable levels of overall locomotor activity (Figure 5L). These results indicate that explorative and anxiety-like behavior are similar in $\mathrm{HCN}^{-/-}$ and wild type mice.

In line with this interpretation in the dark-light test (Figure 5M), another test for exploration-based anxiety, $\mathrm{HCN}^{-/-}$and wild type mice behaved similar. The number of transitions between illuminated and dark chambers was the same in wild type and $\mathrm{HCN}^{-/-}$mice (Figure 5N). Furthermore, both groups of mice strongly avoided the brightly illuminated chamber in favor of the dark compartment (Figure 50). There was no difference in the number of entries into (wild type: 3.87 \pm 0.42 HCN3 $^{-/-}: 3.93 \pm 0.47 ; t=0.1024, p>0.05$ ) and the time spent in the dark compartment (wild type: $79.9 \pm 2.9 \%$; $\mathrm{HCN}^{-/-}: 77.8 \pm 3.1 \%, p>0.05$ ) for both group of mice. Finally, WT and $\mathrm{HCN}^{-/-}$animals showed similar levels of activity in the light area as evident from the total distance mice traveled there (Figure 5P; wild type: $1.97 \pm 0.17 \mathrm{HCN}^{-/-}: 2.24$ $\pm 0.19, t=1.050, p>0.05)$.

We also tested for motor stereotypies, such as repetitive grooming, jumping, and digging, using the marble-burying assay (Figure 6A). For this test, a cage is prepared with a defined layer of wood chip bedding and marbles are placed in a regular pattern on top of the bedding surface. An animal is placed in the cage for $30 \mathrm{~min}$. Thereafter, the mouse is removed, and the number of marbles buried with bedding is counted. Within the 30 -min observation period, $\mathrm{HCN} 3^{-/-}$mice buried a significantly higher number of marbles than wild type $\left(t_{18}=2.933 ; p<\right.$ 0.01 ; unpaired $t$-test; Figure 6A, right). Since we failed to observe changes in anxiety-related behavior on an elevated plus-maze or in the light-dark box (see Figure 5) and general arousal (as assessed by novelty-induced grooming; Figure $\mathbf{5 H}$ ), it is difficult to interpret this finding.

Depressive-like behavior was tested using the forced swimming test (Figure 6B). This test measures the time spent swimming vs. the time spent floating in a tall cylinder filled with water, from which the mice cannot escape. After some time the animal may stop swimming and begin floating. This incidence of passive stress coping is regarded as a sign of behavioral despair. For the test, active (swimming and attempts for climbing) and passive (immobility) behavior is determined and the fraction of floating behavior characterized by passive immobility in relation to total observation time is calculated. Both, wild type and $\mathrm{HCN}^{-/}$mice showed an increase in floating from the first to the second exposure, however, without significant genotype differences [d1-GT: $F_{(1,38)}=0.1583, p>0.05$; d2-GT: $F_{(1,38)}$ $=2.927, p>0.05 ; 2$-way ANOVA for repeated measures; Figure 6B].

Finally, cognitive functions such as learning and memory were tested using the Morris water maze and fear conditioning. To assess functional implications of HCN3 knock-out on spatial memory, we tested mice of both genotypes in the hidden platform version of the Morris water maze (Figure 7A). Mice were trained to locate to a hidden escape platform in a water basin on three consecutive days (navigation training). On day 4 and 5 the reverse hidden platform learning task was performed by moving the platform to the diagonally opposite quadrant.

During water maze training, the parameters escape latencies and swim path length indicate robust learning, reversal effect, and re-learning in both groups of mice, with no difference between the genotypes (Figures 7B,C). There was also no difference in swim speed between the two groups of mice [Genotype: $F_{(1,28)}$ $=2.189, p>0.05$; Supplementary Figure 5], or in any of the other parameters assessed, such as incidence of floating and wall approaches (data not shown). Overall the mice showed little wall hugging, with mutants perhaps tending to spend somewhat more time near the wall [Genotype: $F_{(1,28)}=3.522, p>0.05$; Supplementary Figure 5]. During the probe trial on day 4, mice showed strong preference for the trained quadrant [Place: $F_{(1,28)}=21.34, p<0.001$; Figure 7D]. This was calculated by comparing the time spent in the trained quadrant vs. the averaged time the animals spent in the two adjacent quadrants. Since, during the probe trial, the hidden platform is relocated to 
A
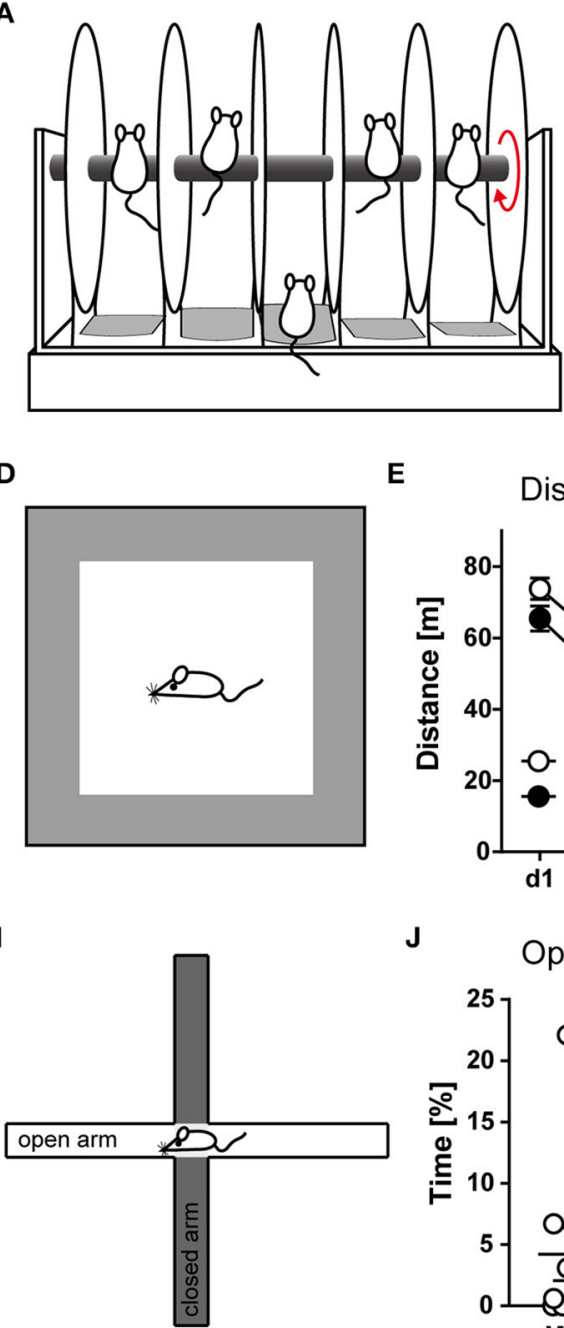

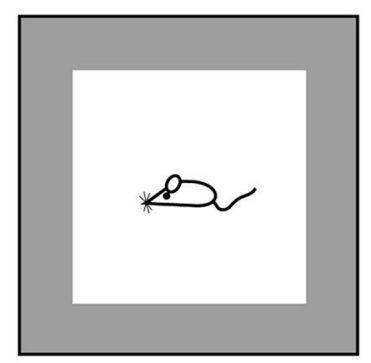

E

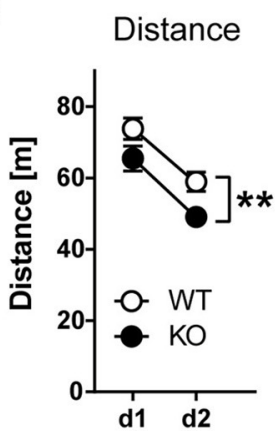

J

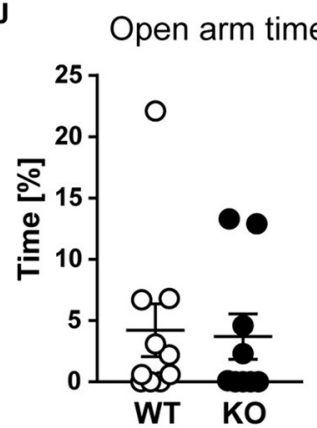

B

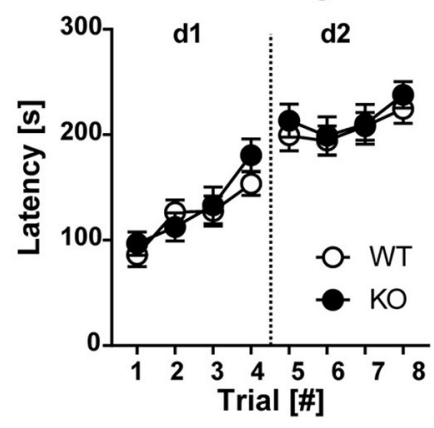

F Immobility
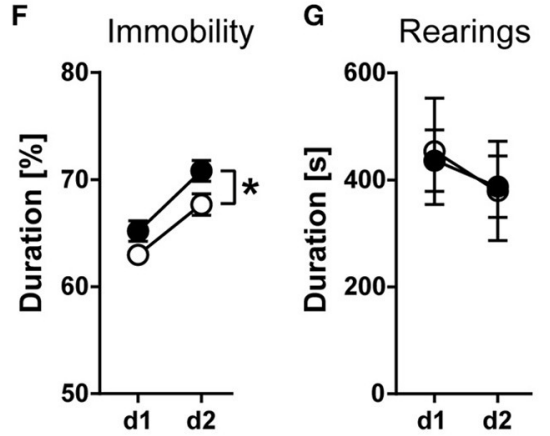

K
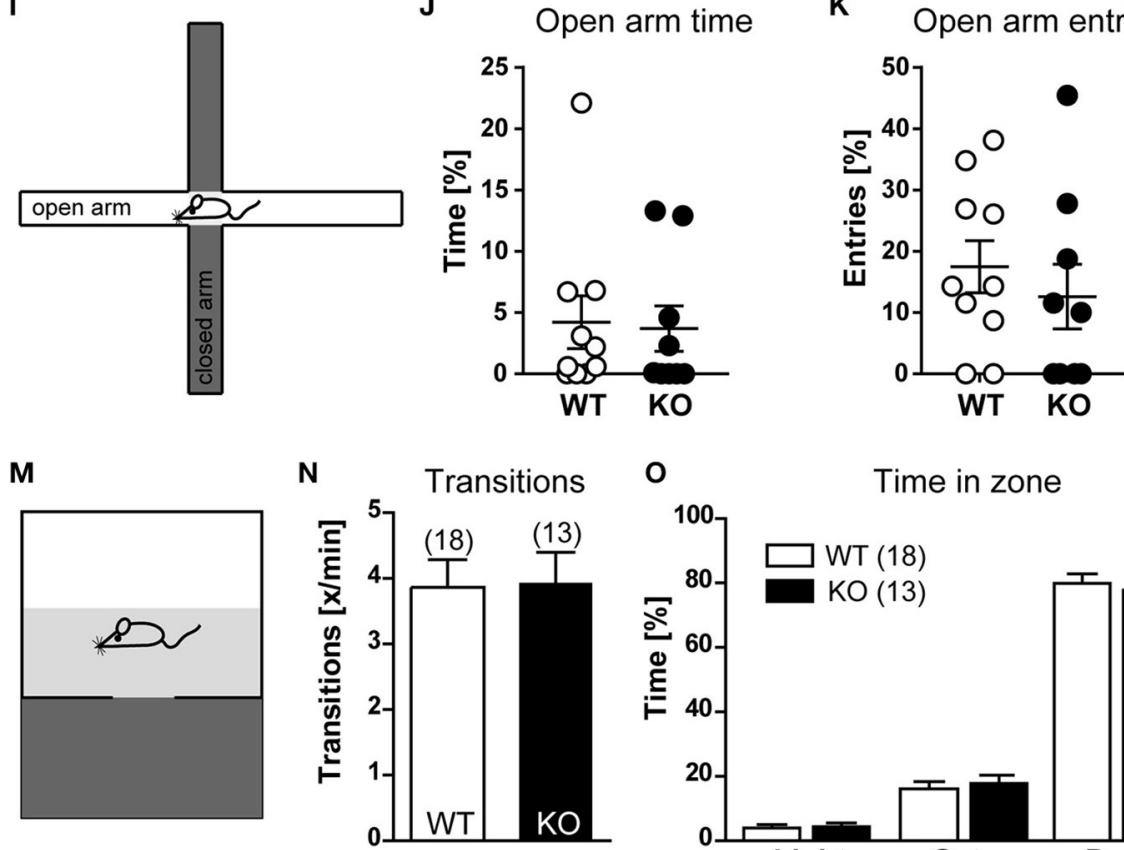

L
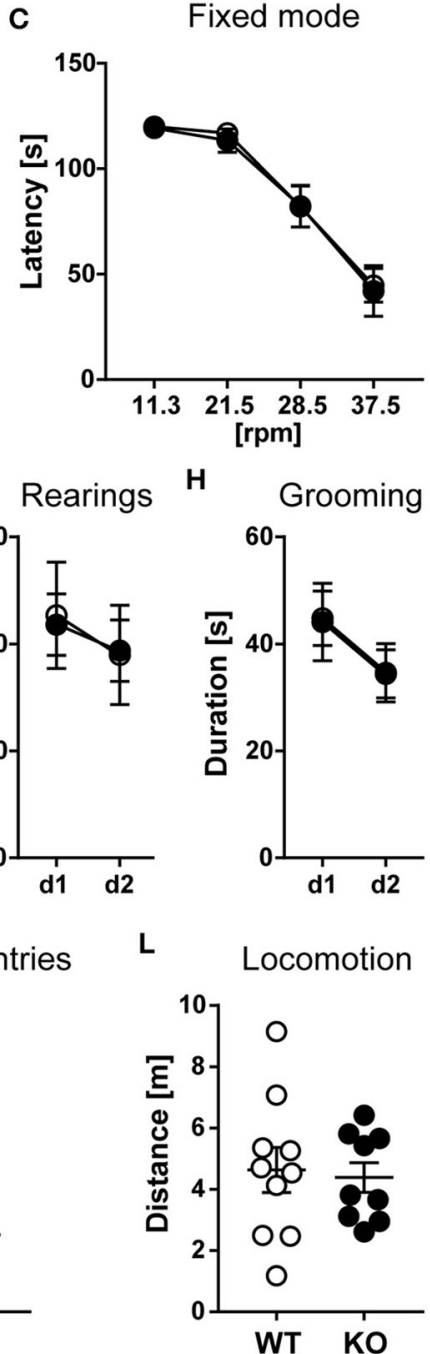

$H$

H Grooming

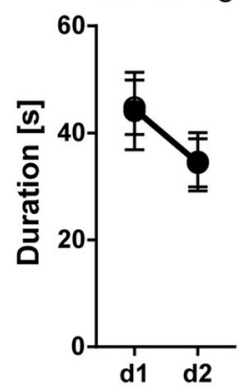

Locomotion

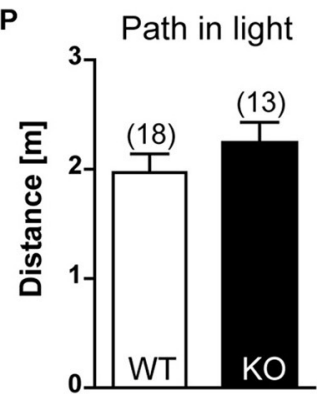

FIGURE 5 | Behavioral characterization of $\mathrm{HCN}^{-/-}$mice (part 1). (A) Schematic of the rotarod test. Mice were placed on drums rotating at an accelerating rotation speed (B) or at the rotation speed indicated (C). The time at which an animal fell from the drum was measured. The increased latency from trial 1 to trial 8 indicates motor learning behavior (B). (D) Schematic of the open field test. (E-H) Open field performance on two consecutive days. Monitoring of general locomotor activity in a novel environment over $10 \mathrm{~min}$. The distance traveled (E), relative time of immobility (F), rearings $(\mathbf{G})$, and grooming $(\mathbf{H})$ are indicated. (I-L) Elevated plus maze. Schematic of the maze (I). Analysis of time spent in open arms (J), entries into open arms (K), and total locomotion (L) revealed no genotype differences. (M) Schematic of the light-dark box. (N-P) In the light-dark box no genotype differences were evident since all animals showed the same number of transitions (N), avoided the brightly illuminated part of the chamber $(\mathbf{O})$, and traveled a similar distance in the bright area (P). All values are Mean \pm SEM, ${ }^{\star} p<0.05,{ }^{\star \star} p<0.01$. 
A

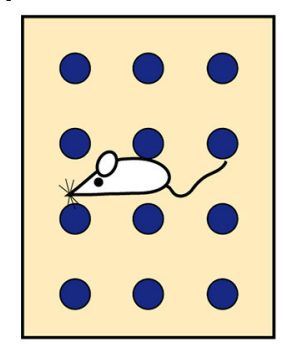

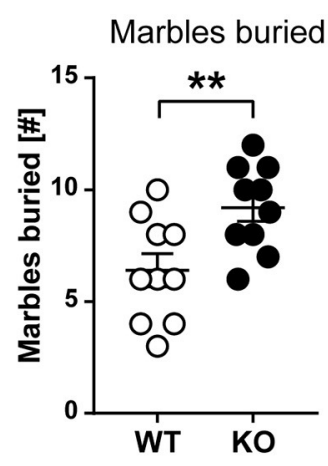

B

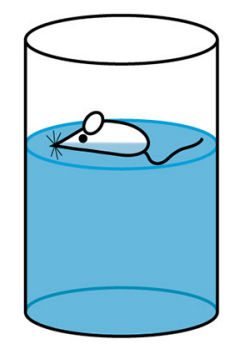

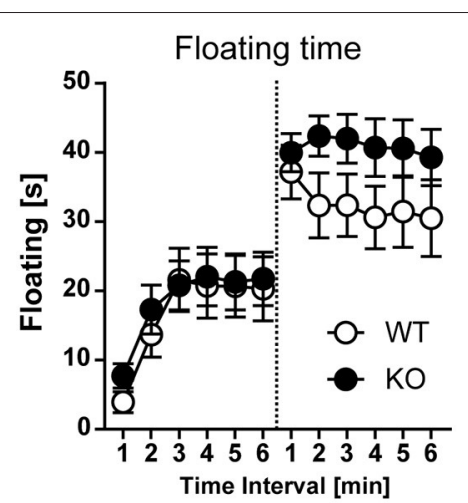

FIGURE 6 | Behavioral characterization of $\mathrm{HCN}^{-/-}$mice (part 2). (A) Marble burying test. Schematic of the testing environment (left), where mice exposed to a cage containing 12 identical glass marbles. $\mathrm{HCN}^{-/-}$mice buried more marbles as compared to wild type littermates during a 30 min exposure time period (right). (B) Forced swimming test. Schematic of the testing environment (left). Mice are placed in an inescapable cylinder filled with water and the time spent floating (immobile) is measured. Mice were exposed to the forced swim test for 6 min on two consecutive days. HCN3 ${ }^{-/}$animals tended to spend more time floating on the second testing day (right). All values are Mean \pm SEM, ${ }^{* \star} p<0.01$

the opposite quadrant, it cannot serve as a neutral control and therefore was not included into the calculation. The preference of $\mathrm{HCN}^{-/-}$mice for the training quadrant was indistinguishable from that of wild type mice [Place $\times$ Genotype: $F_{(1,28)}=0.202, p$ $>0.05$; Figure 7D]. In addition, mice showed a clear preference for a circular zone drawn around the former goal location (12.5\% of pool surface) during the probe trial [Place: $F_{(1,28)}=$ $16.48, p<0.01$; Figure 7E], again with no statistical significant difference between the two genotypes $\left[F_{(1,28)}=1.993, p>\right.$ 0.05 ; Figure $7 \mathrm{E}]$. In summary, the water-maze place navigation task revealed robust learning, reversal effect, re-learning and probe trial retention in both groups, with no significant genotype differences, indicating that $\mathrm{HCN} 3$ channels are not necessary for these processes.

For fear conditioning (Figure $7 \mathbf{F}$ ), $\mathrm{HCN}^{-/-}$, and wild type mice were placed into a shock chamber and after 3 min (pretraining) a tone was presented for $30 \mathrm{~s}$. During the last $2 \mathrm{~s}$ an electric foot shock was applied simultaneously. On day one after conditioning, $\mathrm{HCN}^{-/-}$and wild type animals were placed in a novel chamber representing the neutral context (NC) and were observed for $3 \mathrm{~min}$ (Figure 7F, second panel). Subsequently a tone, but no shock was applied and the mice were observed for additional $3 \mathrm{~min}$ (Figure 7F, third panel). On day two after conditioning, $\mathrm{HCN}^{-/-}$and wild type mice were exposed to the shock chamber (Conditioning context, CC) without applying an electric shock or a tone (Figure 7F, fourth panel). These exposures to neutral and conditioning context were repeated seven days after the first exposure (i.e., on day 7 and 8 after conditioning). Freezing behavior served as a measure of fear. During the 3 mins of pre-training, before presentation of the tone and foot shock, both genotypes exhibited very low and statistically indistinguishable levels of immobility [GT: $F_{(1,18)}=$ $0.7615, p>0.05$; GT $\times$ Time: $F_{(2,36)}=1.034, p>0.05 ; 2$-way ANOVA for repeated measures; data not shown]. Furthermore, $\mathrm{HCN}^{+/+}$and $\mathrm{HCN}^{-/-}$showed the same low levels of freezing during the $3 \mathrm{~min}$ before tone presentation in the novel (neutral) context at d1 [GT: $F_{(1,18)}=0.2399 ; p>0.05 ; \mathrm{GT} \times$ Time:
$F_{(2,36)}=0.0592, p>0.05$; Figure 7G]. During re-exposure to the neutral context at $\mathrm{d} 7$, however, $\mathrm{HCN} 3^{-/}-$showed significantly more basal freezing [GT: $F_{(1,18)}=5.427 ; p<0.05$; Figure 7G]. Presentation of the tone resulted in a strong increase in freezing, both at day 1 and 7 , which was indistinguishable between the two genotypes [Figure 7H; Genotype: $F_{(1,18)} \leq 0.047, p \geq$ $0.830]$. There were also no statistically significant differences in contextual fear (conditional context, without tone presentation or shock application) both at $\mathrm{d} 2\left[\mathrm{GT}: F_{(1,17)}=2.692 ; p>0.05\right]$ and d8 $\left[\mathrm{GT}: F_{(1,17)}=3.792 ; p>0.05\right.$; Figure $\left.7 \mathrm{I}\right]$. In addition, both $\mathrm{HCN}^{+/+}$and $\mathrm{HCN}^{-/-}$showed the same specificity of contextual fear (expressed as the difference in freezing in the conditioning context and the neutral context; $t_{17}=1.415, p>$ 0.05; unpaired $t$-test; Figure 7J).

On closer inspection of short-term and long-term changes in freezing from $\mathrm{d} 1$ to $\mathrm{d} 7$ and $\mathrm{d} 2$ to $\mathrm{d} 8$, respectively, we observed a lack of fear adaption over the course of tone presentation in $\mathrm{HCN}^{-/-}$compared to $\mathrm{HCN}^{+/+}$at $\mathrm{d} 7\left[F_{(2,36)}=4.051\right.$, $p=0.025]$, but not at d1 $\left[F_{(2,36)}=0.506, p=0.606\right]$, as well as striking genotype differences in case of freezing in the neutral context before tone presentation $\left(\mathrm{GT} \times\right.$ Day: $F_{(1,17)}=$ 8.856, $p<0.01$; Figure $7 \mathbf{K})$ and in the conditioning context $\left[\mathrm{GT} \times\right.$ Day: $F_{(1,17)}=7.989 ; p<0.05$; Figure $\left.7 \mathrm{~K}\right]$, but not freezing to the tone $\left[\mathrm{GT} \times\right.$ Day: $F_{(1,18)}=0.1909 ; p>0.05$; Figure $7 \mathbf{K}]$. To emphasize the long-term extinction at the level of individual animals, subsequent analyses separately per genotype confirmed a significant increase in freezing in the neutral context in $\mathrm{HCN}^{-/-}\left(t_{9}=3.564, p<0.01\right.$; paired $t$-test; Figure $\left.7 \mathrm{~L}\right)$, but not $\mathrm{HCN}^{+/+}$mice $\left(t_{9}=0.938, p>0.05\right.$; Figure $\left.7 \mathrm{~L}\right)$, whereas freezing to the tone was decreased in $\mathrm{HCN}^{-/-}$and tended to be decreased in $\mathrm{HCN}^{+/+}$animals (Figure $7 \mathbf{M}$ ). In contrast, $\mathrm{HCN}^{+/+}\left(t_{9}=3.205, p=0.01\right.$; Figure $\left.7 \mathbf{N}\right)$, but not $\mathrm{HCN}^{-/-}$ $\left(t_{8}=0.329, p>0.05\right.$; Figure $\left.7 \mathbf{N}\right)$, showed a significant decrease in contextual fear from $\mathrm{d} 2$ to $\mathrm{d} 8$. In summary, $\mathrm{HCN} 3^{-/}$showed deficits in acute fear adaption upon re-exposure to the tone and long-term processing of contextual fear, which can be interpreted as lack of extinction and increase in context generalization. 

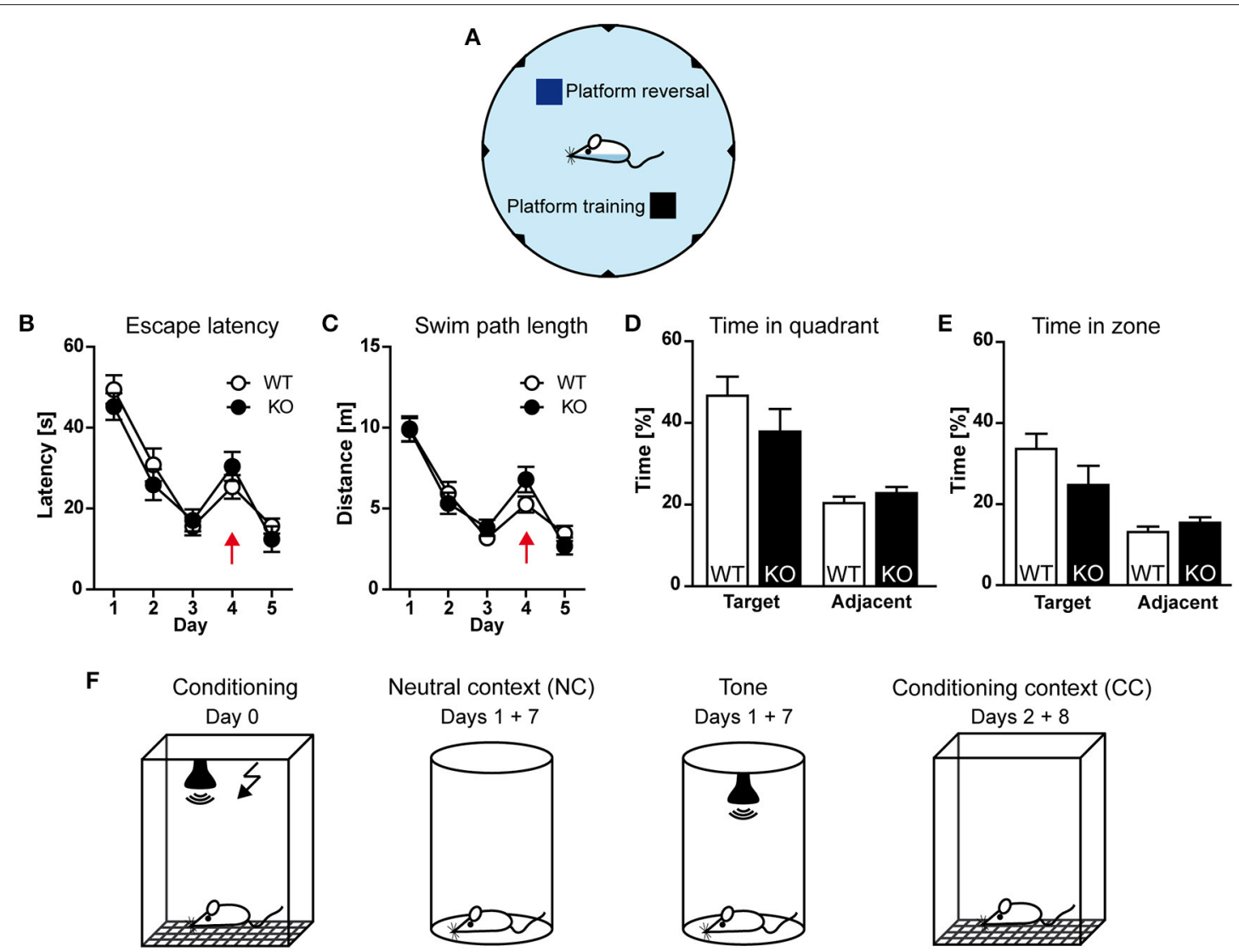

Conditioning context (CC)
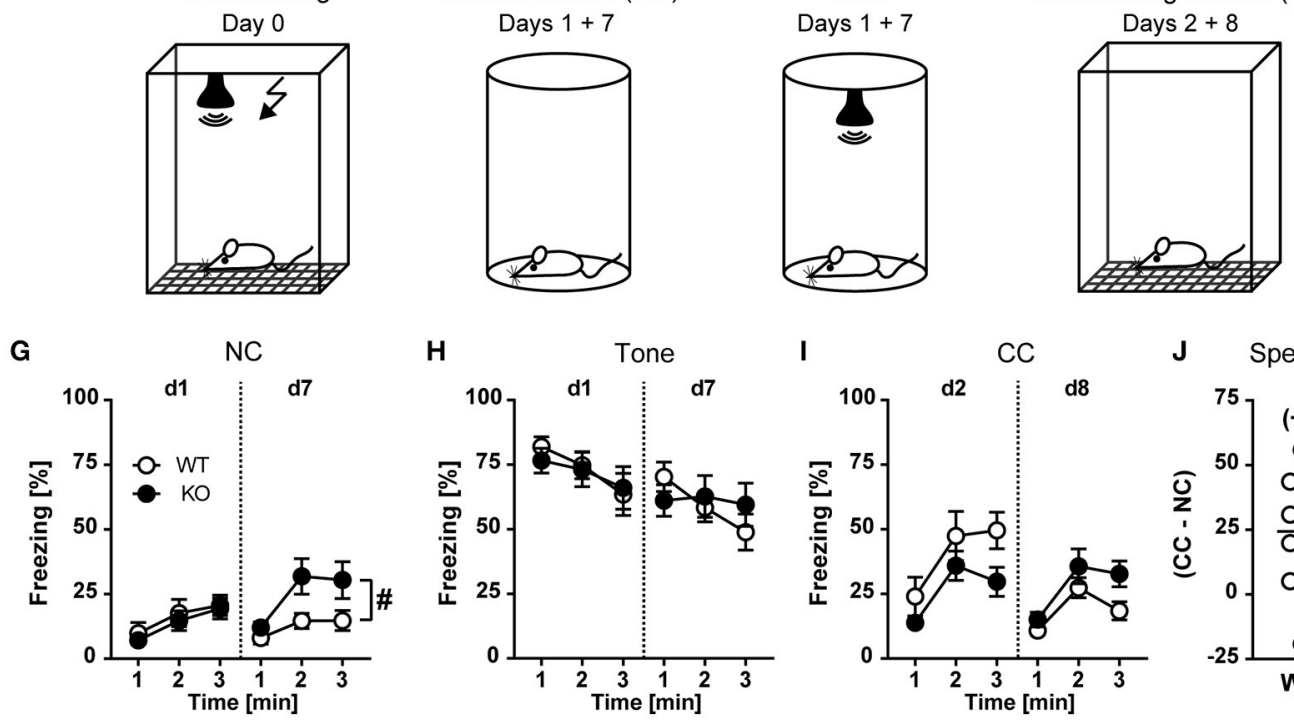

J Specificity (d1)
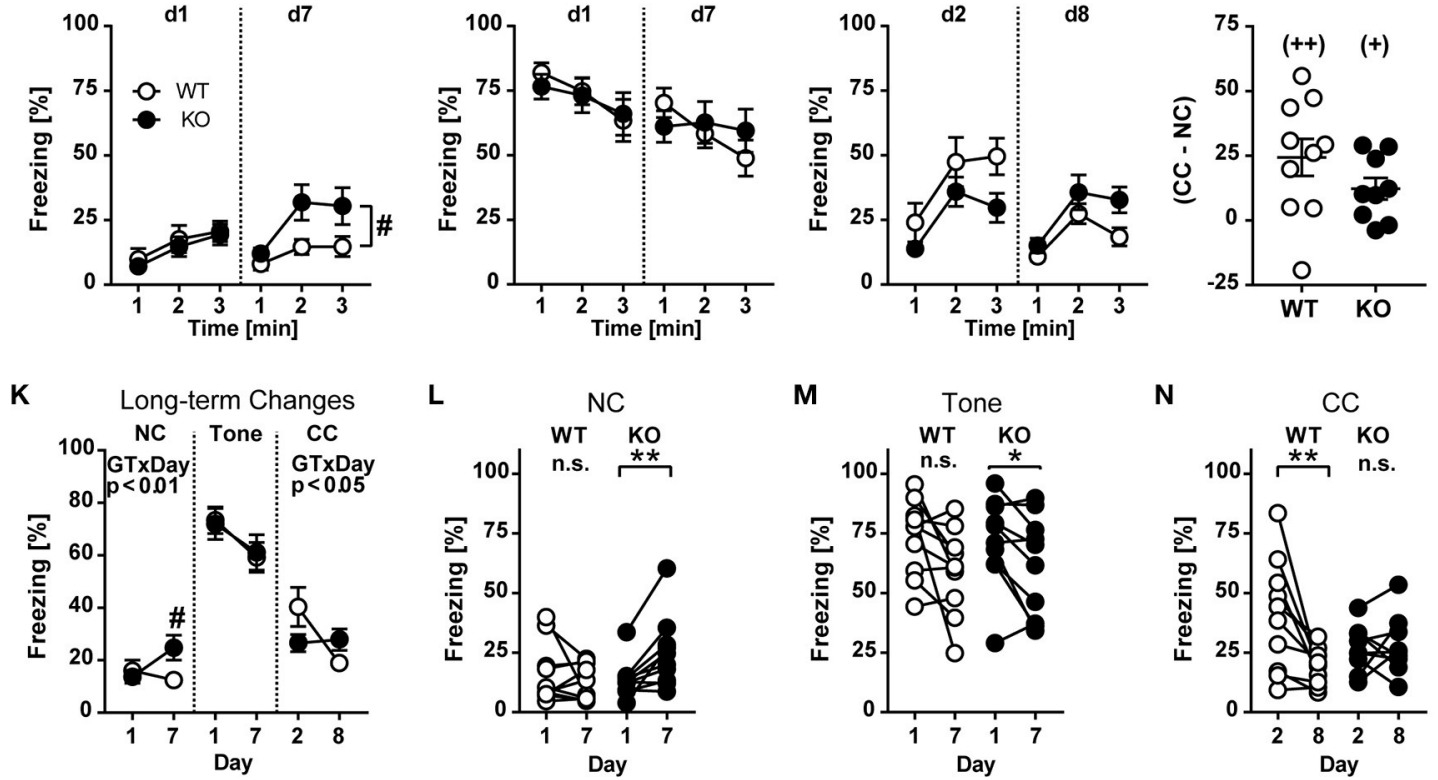

FIGURE 7 | Behavioral characterization of $\mathrm{HCN}^{-/-}$mice (part 3). (A-E) Morris water maze. During training (days 1-3) the platform was hidden in a specific quadrant of the maze and its position was changed on the first trial of day 4 (probe trial) to a different quadrant and remained there until the end of the experiment (A). According to escape latencies (B) and swim path length (C), mice showed robust learning (days 1-3), reversal effect (day 4; indicated by red arrow), and re-learning (day 5) with no evidence for a genotype effect. During the probe trial (first trial on day 4) the mice showed a robust preference for the trained quadrant compared to the average of the two adjacent quadrants (D) and for a circular zone (12.5\% of pool surface) drawn around the former platform location (E). (F-N) Fear conditioning. For 
FIGURE 7 | conditioning, mice were placed into a shock chamber and a tone was presented followed by an electric foot shock (F, first panel). On d1 and d7 after conditioning, mice were observed in the neutral context before (NC) and during subsequent exposure of the tone (tone) for 3 min each, and to the shock chamber (CC) on $\mathrm{d} 2$ and $\mathrm{d} 8$ without applying an electric shock or a tone ( 3 min each). (G) Freezing in the neutral context during the 3 min before tone presentation (expressed in 1-min intervals) at d1 and d7. (H) Freezing to the tone at d1 and d7 (1-min intervals). (I) Freezing in the conditioning context at d1 and d7 (1-min intervals). (J) Specificity of contextual fear expressed as the difference between freezing in the CC and the NC. (K) Long-term changes in freezing from d1 to d7. (L-N) Individual data plots depicting long-term changes in freezing from d1 to d7 separately per genotype. ${ }^{*} p<0.05,{ }^{* \star} p<0.01$ (paired $t$-test), ${ }^{+} p<0.05$, ${ }^{++} p<0.01$ (one-sample $t$-test against theoretical value 0), $\# p<0.05$ (KO vs. WT; 2-way ANOVA followed by Bonferroni post-hoc test, if appropriate). One KO was excluded from further analysis of contextual fear since it was identified as outlier (http://www.graphpad.com/quickcalcs/grubbs1/).

\section{DISCUSSION}

The functional role of HCN1 and HCN2 channels have been extensively analyzed in the heart and the brain. While for all members of the HCN channel family genetic knockout mouse models are available, the neuronal phenotype of $\mathrm{HCN}^{-/-}$mice has not been investigated and reported so far. In this study, we have used $\mathrm{HCN}^{-/-}$mice to investigate the specific physiological role of this channel in the CNS. Here, we specifically focused on the significance of this channel for circadian rhythm and behavior.

Using the $\mathrm{HCN}^{-/-}$mouse line, we have previously reported the role of HCN3 channels for late repolarization in action potential of ventricular cardiomyocytes (Fenske et al., 2011). A comprehensive characterization of the $\mathrm{HCN}^{-/-}$mouse revealed that there are no obvious other functional organ specific phenotypes. In particular liver function tests, exocrine, and endocrine pancreatic function, homeostasis of electrolytes and regulation of plasma lipid and lipoprotein levels were normal. Also, body weight was unaffected. However, we found that $\mathrm{Ca}^{2+}$ and Urea levels were significantly decreased in $\mathrm{HCN}^{-/-}$animals. Since HCN3 is expressed in kidneys of several species, the question arises whether these findings could represent a renal phenotype, or might be caused by extrarenal metabolic or humoral changes. This issue needs to be addressed in future studies. Furthermore, we did not find any evidence for a functional role of these channels for the regulation of hypothalamus-dependent release of corticotropin-releasing factor, and corticosterone. This indicates that HCN3 channels do not seem to be functionally relevant for regulation of brain CRF or plasma glucocorticoid levels under baseline conditions. Nevertheless, due to their high expression in the PVN, HCN3 channels might still be implicated in the stress response provoked by acute or chronic stressors, which needs to be verified in future experiments. Furthermore, there was no change in $\mathrm{ADH}$ dependent electrolyte or water balance, indicating that $\mathrm{HCN} 3$ channels in $\mathrm{ADH}$ positive cells in the hypothalamic region are not critical for regulating plasma electrolyte composition under baseline conditions. We then focused in particular on the role of HCN3 in the circadian system and in the control of behavior.

\section{Circadian System}

To analyze the function of $\mathrm{HCN} 3$ channels in the major network regulating the circadian rhythm (Figure 1) we studied expression and function of this channel in the SCN, the retina, and in the IGL. Surprisingly there is no clear expression of
HCN3 in the SCN. Our own results as well as data from the literature (Notomi and Shigemoto, 2004) suggest that in the SCN, other HCN channels may be present, most likely HCN4. The role of this channel in controlling circadian behavior is not known.

In the retina, $\mathrm{HCN} 3$ channel labeling was restricted to the outer and inner plexiform layers of the retina, which harbor the synapses of photoreceptor and bipolar neurons, respectively. This finding is in line with a previous report, which also found HCN3 expression in the outer plexiform layer (Muller et al., 2003). The authors of this study concluded that HCN3 is anatomically restricted to the base of cone pedicle in the outer plexiform layer and suggested that HCN channels might directly modulate synaptic transmission of photoreceptor activity to bipolar cells (Muller et al., 2003), which was indirectly tested in the present study. Surprisingly, our electrophysiological results did not confirm this appealing hypothesis. In fact, retinal function testing in $\mathrm{HCN}^{-/-}$animals indicated normal cone and rod function under both photopic and scotopic conditions. In particular, the b-wave was normal in $\mathrm{HCN}^{-/-}$ mice, which directly reflects the activity of bipolar cells in the inner retina and indirectly reflects activity of photoreceptors and successful synaptic transmission at the first synapse. A normal, unreduced b-wave therefore indicates regular activity in these synapses and argues against a major role of HCN3 channels for transmission in photoreceptor synapses including those of cone pedicles. Given the relatively large deactivation time constants of HCN3 channels (Fenske et al., 2011) it would be possible that $\mathrm{HCN} 3$ channels are involved in slower steps of visual information processing in the retina. One possible scenario would be that HCN3 is involved in long-term adaptation and thus complement the role of HCN1 channels in shortterm adaptation of visual function. HCN1 channels have been shown to be predominantly located in the inner segments of rod and cone photoreceptors (Seeliger et al., 2011). These channels prevent saturation of the retinal network following bright light exposure, prevent prolonged "blinding" by light and speed up regain of vision. Beside $\mathrm{HCN} 1$ and $\mathrm{HCN} 3$ channels, HCN4 channels are present in the retina (Muller et al., 2003). The function of this channel is not known. In conclusion, retinal function is completely normal in $\mathrm{HCN}^{-/-}$mice. Furthermore, the flow of photic information from the retina to the SCN and IGL via the retinohypothalamic tract, which is critical for resetting of the circadian clock, is also normal, even in the absence of $\mathrm{HCN} 3$.

We found high levels of HCN3 protein expression in the IGL. This finding is in line with reports from Ying et al. 
(2011). In addition, we find evidence for different populations of neurons in the IGL, one population in which $\mathrm{I}_{\mathrm{h}}$ is completely absent in $\mathrm{HCN}^{-/-}$neurons, and a second population in which a remaining $\mathrm{I}_{\mathrm{h}}$ is present. Consistent with our IHC experiments this remaining $\mathrm{I}_{\mathrm{h}}$ is most likely conferred by HCN2. The IGL has been demonstrated to be an important nucleus upstream of the SCN, which integrates photic and non-photic information (Delogu et al., 2012) and eventually implicates circadian behavior via output firing to the SCN. In contrast to these hypotheses, our results indicate that HCN3 channels do not play a major role in the regulation of circadian locomotor behavior under constant lighting conditions. Furthermore, we could not confirm a major role of these channels for light induced resetting mechanism of the circadian clock. Moreover, HCN3 channels do not play an important role in the speed of adaptation in the behavior of mice to a new LD cycle. However, in the absence of a light cue [constant darkness conditions (DD)] $\mathrm{HCN3}^{-/-}$animals display less activity (Figure 4C). This result suggests that HCN3 is important for reinforcing locomotor activity under DD conditions. Together the circadian experiments indicate that even if the HCN3 component of $\mathrm{I}_{\mathrm{h}}$ is responsible for rhythmic oscillations formed by spontaneous low threshold spike burst firing of pacemaker neurons in the IGL as suggested by Ying et al., this change is without consequence for IGL dependent modulation of the SCN and SCN dependent circadian behavior in the presence of light cues. This may be different in the absence of light cues as observed under DD conditions. In conclusion, although HCN3 channels are highly expressed in the IGL, their deletion does not significantly alter photic, IGL-specific regulation of circadian function. However, we cannot completely rule out the possibility that in $\mathrm{HCN3}^{-/-}$animals the compensatory upregulation of other $\mathrm{HCN}$ channel family members might mask a possible phenotype. Given that in (Fenske et al., 2011) no such upregulation was found in microarray experiments this possibility is rather unlikely.

\section{Behavior}

Given the broad intracerebral expression of the HCN3 channel, behavioral consequences of its functional knock-out were remarkably distinct: Except for a reduction in horizontal locomotion, we failed to observe genotype effects in a variety of tests assessing motor learning and motor coordination (rotarod), exploration-based anxiety-related behavior (elevated plus maze, dark-light transition test), behavioral stress coping (forced swim test), and hippocampus-(Morris water maze, acquisition, and consolidation of contextual fear) and amygdala-dependent learning (auditory-cued fear conditioning). In contrast, $\mathrm{HCN}^{-/-}$showed enhanced marble burying and impaired processing of contextual fear upon repeated (re)exposure to the conditioning and the neutral test context: specifically, whereas wild type mice showed extinction of contextual fear from $\mathrm{d} 2$ to $\mathrm{d} 8, \mathrm{HCN}^{-/-}$failed to do so. In contrast, $\mathrm{HCN}^{-/-}$, but not wild type mice, developed generalized fear to the neutral context from $\mathrm{d} 1$ to $\mathrm{d} 7$. Here, the process of picking up of the animals by the tail itself and placing them into the test environment might be sufficient to trigger "context generalization." At the same time, we cannot rule out whether the failure to decrease freezing behavior might be ascribed to second-order conditioning. In this type of conditioning, the animals learn to associate the neutral context with the aversive tone during testing at day 1. Alternatively, an unspecific increase in general anxiety could explain the behavioral effects. In contrast, the observed alterations cannot be explained by general changes in locomotor activity or long-term habituation, since both genotypes showed the same decrease in locomotion over two consecutive exposures to an open field. Another factor that might influence the outcome of the fear conditioning paradigm are changes in auditory function. While we did not perform in depth analysis of the auditory function in $\mathrm{HCN}^{-/-}$mice, we can exclude a major overall auditory phenotype in these mice, because freezing behavior upon presentation of the tone was similar in wild type and $\mathrm{HCN}^{-/-}$animals. This finding indicates that even though we cannot rule out subtle differences in auditory function between $\mathrm{HCN}^{-/-}$and WT mice, these differences, if present, do not interfere with the fear conditioning paradigm and our conclusions. Finally, a change in pain perception per se could account for changes in fear conditioning. It has been shown that $\mathrm{HCN}$ channels are expressed in dorsal root ganglia of mice and rat (Kouranova et al., 2008; Hou et al., 2015; Usoskin et al., 2015). There is also evidence that HCN2 channels might be involved in the regulation of neuropathic and inflammatory pain (Emery et al., 2011). By contrast, there is currently no evidence that HCN channels are involved in acute somatosensory nociceptive pain. In line with this notion, the study by Emery et al. (2011) showed that pain reactions toward acute mechanical and heat stimuli were not affected after application of ZD7288, a non-selective blocker of HCN channels. This result indicates that even though HCN3 channels might be expressed in dorsal root ganglia, these channels are not involved in fundamental regulation of acute somatosensory nociceptive pain, e.g. the subtype of pain relevant in fear conditioning experiments.

Currently we can only speculate about the biological processes underlying the increase in marble burying and fear generalization and the impairment in fear extinction. Given (i) the close correlation between pharmacologically enhanced monoamine levels within the mPFC and alterations in marble burying (increased levels of serotonin correlated positively with inhibition of marble burying, whereas changes in noradrenaline revealed the reverse relationship; (Kobayashi et al., 2008), (ii) the role of the mPFC in fear extinction (for review see Maren et al., 2013), and (iii) the implication of mPFC projections to the thalamus in generalization of contextual fear ( $\mathrm{Xu}$ and Sudhof, 2013), it is tempting to assume a contribution of corticothalamic projections to the observed phenotype. In line with this hypothesis, HCN channels seem to be present in dendritic spines of mPFC neurons (Wang et al., 2007). Furthermore, HCN3 channels are expressed in the basolateral amygdala and the hippocampus in the rat (Notomi and Shigemoto, 2004) and mouse brains (own observations). These brain regions are implicated in the formation of associative memory, learning and expression of fear. On the cellular level, HCN channels have been associated with a variety of 
physiological roles in the CNS, such as setting of the resting membrane potential and therefore controlling the excitability of a given neuron, or synaptic integration and plasticity. Hence, deletion of $\mathrm{HCN} 3$ might alter the intrinsic activity of neurons in the amygdala complex or hippocampal neurons and disturb the proper information processing of learned fear. Future studies will reveal the details how exactly HCN3 channels in these neurons are involved in regulation of fear generalization.

\section{AUTHOR CONTRIBUTIONS}

MS performed experiments, analyzed data and wrote the manuscript. SF, VH, and EB provided guidance and designed experiments. VS, MS-W, RM, and JD performed experiments and analyzed data. JED and UA planned and performed experiments, analyzed data, and helped to write the manuscript. DW planned and performed experiments and analyzed data, MWS planned experiments, analyzed data and assisted in writing the manuscript. CW and SM provided guidance, analyzed data and assisted to write the manuscript. MB carried out the study design, provided guidance and assisted to write the manuscript.

\section{REFERENCES}

Albrecht, U. (2012). Timing to perfection: the biology of central and peripheral circadian clocks. Neuron 74, 246-260. doi: 10.1016/j.neuron.2012.04.006

Arroyo, A., Kim, B., Rasmusson, R. L., Bett, G., and Yeh, J. (2006). Hyperpolarization-activated cation channels are expressed in rat hypothalamic gonadotropin-releasing hormone $(\mathrm{GnRH})$ neurons and immortalized $\mathrm{GnRH}$ neurons. J. Soc. Gynecol. Investig. 13, 442-450. doi: 10.1016/j.jsgi.2006.05.010

Aschoff, J. C. (1977). Sulpiride in the prevention of migraine. A mechanism of hormonal interaction? Fortschr Med. 95, 523-527.

Baruscotti, M., Bottelli, G., Milanesi, R., DiFrancesco, J. C., and DiFrancesco, D. (2010). HCN-related channelopathies. Pflugers Arch. 460, 405-415. doi: 10.1007/s00424-010-0810-8

Battefeld, A., Rocha, N., Stadler, K., Brauer, A. U., and Strauss, U. (2012). Distinct perinatal features of the hyperpolarization-activated nonselective cation current $\mathrm{I}(\mathrm{h})$ in the rat cortical plate. Neural Dev. 7:21. doi: 10.1186/1749-8104-7-21

Biel, M., Wahl-Schott, C., Michalakis, S., and Zong, X. (2009). Hyperpolarizationactivated cation channels: from genes to function. Physiol. Rev. 89, 847-885. doi: 10.1152 /physrev.00029.2008

Castagné, V., Moser, P., Roux, S., and Porsolt, R. D. (2011). Rodent models of depression: forced swim and tail suspension behavioral despair tests in rats and mice. Curr. Protoc. Neurosci. Chapter 8: Unit 8.10A. doi: 10.1002/0471142301.ns0810as55

Crawley, J., and Goodwin, F. K. (1980). Preliminary report of a simple animal behavior model for the anxiolytic effects of benzodiazepines. Pharmac. Biochem. Behav. 13, 167-170. doi: 10.1016/0091-3057(80)90067-2

Deacon, R. M. J. (2006). Digging and marble burying in mice: simple methods for in vivo identification of biological impacts. Nat. Protoc. 1, 122-124. doi: 10.1038 /nprot.2006.20

Delogu, A., Sellers, K., Zagoraiou, L., Bocianowska-Zbrog, A., Mandal, S., Guimera, J., et al. and Lumsden, A. (2012). Subcortical visual shell nuclei targeted by ipRGCs develop from a Sox14+-GABAergic progenitor and require Sox14 to regulate daily activity rhythms. Neuron 75, 648-662. doi: 10.1016/j.neuron.2012.06.013

Emery, E. C., Young, G. T., Berrocoso, E. M., Chen, L., and McNaughton, P. A. (2011). HCN2 ion channels play a central role in inflammatory and neuropathic pain. Science 333, 1462-1466. doi: 10.1126/science.1206243
CW-S carried out the study design and wrote the manuscript. All authors have read and approved the final version of the manuscript.

\section{FUNDING}

This work was supported by the Deutsche Forschungsgemeinschaft SFB 870. The funders had no role in study design, data collection and analysis, decision to publish, or preparation of the manuscript.

\section{ACKNOWLEDGMENTS}

We thank Dr. Barthelmes and Dr. Schulze for sharing their expertise.

\section{SUPPLEMENTARY MATERIAL}

The Supplementary Material for this article can be found online at: https://www.frontiersin.org/articles/10.3389/fnmol. 2017.00436/full\#supplementary-material

Fenske, S., Mader, R., Scharr, A., Paparizos, C., Cao-Ehlker, X., Michalakis, S., et al. (2011). HCN3 contributes to the ventricular action potential waveform in the murine heart. Circ. Res. 109, 1015-1023. doi: 10.1161/CIRCRESAHA.111.246173

Franklin, K. B. J., and Paxinos, G. (2007). The Mouse Brain in Stereotaxic Coordinates. San Diego, CA: Elsevier Academic Press

Fritz, A., Amrein, I., and Wolfer, D. P. (2017). Similar reliability and equivalent performance of female and male mice in the open-field and water-maze place navigation task. Am. J. Med. Genet. 175, 380-391 doi: 10.1002/ajmg.c.31565

Giocomo, L. M., Hussaini, S. A., Zheng, F., Kandel, E. R., Moser, M. B., and Moser, E. I. (2011). Grid cells use HCN1 channels for spatial scaling. Cell 147, 1159-1170. doi: 10.1016/j.cell.2011.08.051

Hou, B., Chen, H., Qu, X., Lin, X., Luo, F., and Li, C. (2015). Characteristics of hyperpolarization-activated cyclic nucleotide-gated channels in dorsal root ganglion neurons at different ages and sizes. Neuroreport 26, 981-987. doi: 10.1097/WNR.0000000000000455

Hussaini, S. A., Kempadoo, K. A., Thuault, S. J., Siegelbaum, S. A., and Kandel, E. R. (2011). Increased size and stability of CA1 and CA3 place fields in HCN1 knockout mice. Neuron 72, 643-653. doi: 10.1016/j.neuron.2011. 09.007

Jud, C., Schmutz, I., Hampp, G., Oster, H., and Albrecht, U. (2005). A guideline for analyzing circadian wheel-running behavior in rodents under different lighting conditions. Biol. Proced. Online 7, 101-116. doi: 10.1251/bpo109

Kamprath, K., and Wotjak, C. T. (2004). Nonassociative learning processes determine expression and extinction of conditioned fear in mice. Learn. Mem. 11, 770-786. doi: 10.1101/lm.86104

Kanyshkova, T., Meuth, P., Bista, P., Liu, Z., Ehling, P., Caputi, L., et al. (2012). Differential regulation of HCN channel isoform expression in thalamic neurons of epileptic and non-epileptic rat strains. Neurobiol. Dis. 45, 450-461. doi: 10.1016/j.nbd.2011.08.032

Kanyshkova, T., Pawlowski, M., Meuth, P., Dube, C., Bender, R. A., Budde, T., et al. (2009). Postnatal expression pattern of HCN channel isoforms in thalamic neurons: relationship to maturation of thalamocortical oscillations. J. Neurosci. 29, 8847-8857. doi: 10.1523/JNEUROSCI.0689-09.2009

Kim, C. S., Chang, P. Y., and Johnston, D. (2012). Enhancement of dorsal hippocampal activity by knockdown of HCN1 channels leads to anxiolytic- and antidepressant-like behaviors. Neuron 75, 503-516. doi: 10.1016/j.neuron.2012.05.027 
Kobayashi, T., Hayashi, E., Shimamura, M., Kinoshita, M., and Murphy, N. P. (2008). Neurochemical responses to antidepressants in the prefrontal cortex of mice and their efficacy in preclinical models of anxiety-like and depressionlike behavior: a comparative and correlational study. Psychopharmacology 197, 567-580. doi: 10.1007/s00213-008-1070-6

Kouranova, E. V., Strassle, B. W., Ring, R. H., Bowlby, M. R., and Vasilyev, D. V. (2008). Hyperpolarization-activated cyclic nucleotide-gated channel mRNA and protein expression in large versus small diameter dorsal root ganglion neurons: correlation with hyperpolarization-activated current gating. Neuroscience 153, 1008-1019. doi: 10.1016/j.neuroscience.2008.03.032

Kretschmannova, K., Kucka, M., Gonzalez-Iglesias, A. E., and Stojilkovic, S. S. (2012). The expression and role of hyperpolarization-activated and cyclic nucleotide-gated channels in endocrine anterior pituitary cells. Mol. Endocrinol. 26, 153-164. doi: 10.1210/me.2011-1207

Leist, M., Datunashvilli, M., Kanyshkova, T., Zobeiri, M., Aissaoui, A., Cerina, M., et al. (2016). Two types of interneurons in the mouse lateral geniculate nucleus are characterized by different h-current density. Sci. Rep. 6:24904. doi: $10.1038 /$ srep 24904

Leitinger, B., Poletaeva, I. I., Wolfer, D. P., and Lipp, H. (1994). Swimming navigation, open-field activity, and extrapolation behavior of two inbred mouse strains with robertsonian translocation of chromosomes 8 and 17. Behav. Genet. 24, 273-284. doi: 10.1007/BF01067194

Maren, S., Phan, K. L., and Liberzon, I. (2013). The contextual brain: implications for fear conditioning, extinction and psychopathology. Nat. Rev. Neurosci. 14, 417-428. doi: 10.1038/nrn3492

McCormick, D. A., and Bal, T. (1997). Sleep and arousal: thalamocortical mechanisms. Annu. Rev. Neurosci. 20, 185-215. doi: 10.1146/annurev.neuro.20.1.185

Mistrik, P., Mader, R., Michalakis, S., Weidinger, M., Pfeifer, A., and Biel, M. (2005). The murine HCN3 gene encodes a hyperpolarization-activated cation channel with slow kinetics and unique response to cyclic nucleotides. J. Biol. Chem. 280, 27056-27061. doi: 10.1074/jbc.M502696200

Morris, R. (1984). Developments of a water-maze procedure for studying spatial learning in the rat. J. Neurosci. Methods 11, 47-60.

Muller, F., Scholten, A., Ivanova, E., Haverkamp, S., Kremmer, E., and Kaupp, U. B. (2003). HCN channels are expressed differentially in retinal bipolar cells and concentrated at synaptic terminals. Eur. J. Neurosci. 17, 2084-2096. doi: 10.1046/j.1460-9568.2003.02634.x

Nolan, M. F., Malleret, G., Dudman, J. T., Buhl, D. L., Santoro, B., Morozov, A., et al. (2004). A behavioral role for dendritic integration: HCN1 channels constrain spatial memory and plasticity at inputs to distal dendrites of CA1 pyramidal neurons. Cell 119, 719-732. doi: 10.1016/j.cell.2004.11.020

Nolan, M. F., Malleret, G., Lee, K. H., Gibbs, E., Dudman, J. T., Morozov, A., et al. (2003). The hyperpolarization-activated HCN1 channel is important for motor learning and neuronal integration by cerebellar Purkinje cells. Cell 115, 551-564. doi: 10.1016/S0092-8674(03)00884-5

Notomi, T., and Shigemoto, R. (2004). Immunohistochemical localization of Ih channel subunits, HCN1-4, in the rat brain. J. Comp. Neurol. 471, 241-276. doi: $10.1002 /$ cne. 11039

Pape, H. C. (1996). Queer current and pacemaker: the hyperpolarizationactivated cation current in neurons. Annu. Rev. Physiol. 58, 299-327. doi: 10.1146/annurev.ph.58.030196.001503

Pellow, S., and File, S. E. (1986). Anxiolytic and anxiogenic drug effects on exploratory activity in an elevated plus-maze: a novel test of anxiety in the rat. Pharmacol. Biochem. Behav. 24, 525-529. doi: 10.1016/0091-3057(86)90552-6

Peng, S. C., Wu, J., Zhang, D. Y., Jiang, C. Y., Xie, C. N., and Liu, T. (2017). Contribution of presynaptic HCN channels to excitatory inputs of spinal substantia gelatinosa neurons. Neuroscience 358, 146-157. doi: 10.1016/j.neuroscience.2017.06.046
Rathkolb, B., Decker, T., Fuchs, E., Soewarto, D., Fella, C., Heffner, S., et al. (2000). The clinical-chemical screen in the Munich ENU mouse mutagenesis project: screening for clinically relevant phenotypes. Mamm. Genome 11, 543-546. doi: 10.1007/s003350010104

Reid, C. A., Phillips, A. M., and Petrou, S. (2012). HCN channelopathies: pathophysiology in genetic epilepsy and therapeutic implications. $\mathrm{Br} . \mathrm{J}$. Pharmacol. 165, 49-56. doi: 10.1111/j.1476-5381.2011.01507.x

Robinson, R. B., and Siegelbaum, S. A. (2003). Hyperpolarization-activated cation currents: from molecules to physiological function. Annu. Rev. Physiol. 65, 453-480. doi: 10.1146/annurev.physiol.65.092101. 142734

Seeliger, M. W., Beck, S. C., Pereyra-Munoz, N., Dangel, S., Tsai, J. Y., Luhmann, U. F., et al. (2005). In vivo confocal imaging of the retina in animal models using scanning laser ophthalmoscopy. Vis. Res. 45, 3512-3519. doi: 10.1016/j.visres.2005.08.014

Seeliger, M. W., Brombas, A., Weiler, R., Humphries, P., Knop, G., Tanimoto, N., et al. (2011). Modulation of rod photoreceptor output by HCN1 channels is essential for regular mesopic cone vision. Nat. Commun. 2:532. doi: 10.1038/ncomms1540

Shah, M. M., Anderson, A. E., Leung, V., Lin, X., and Johnston, D. (2004). Seizure-induced plasticity of $\mathrm{h}$ channels in entorhinal cortical layer III pyramidal neurons. Neuron 44, 495-508. doi: 10.1016/j.neuron.2004. 10.011

Shah, M. M., Huang, Z., and Martinello, K. (2013). HCN and KV7 (M-) channels as targets for epilepsy treatment. Neuropharmacology 69, 75-81. doi: 10.1016/j.neuropharm.2012.03.005

Tanimoto, N., Muehlfriedel, R. L., Fischer, M. D., Fahl, E., Humphries, P., Biel, M., et al. (2009). Vision tests in the mouse: functional phenotyping with electroretinography. Front. Biosci. 14, 2730-2737. doi: 10.2741/3409

Usoskin, D., Furlan, A., Islam, S., Abdo, H., Lonnerberg, P., Lou, D., et al. (2015). Unbiased classification of sensory neuron types by large-scale single-cell RNA sequencing. Nat. Neurosci. 18, 145-153. doi: 10.1038/nn.3881

Wang, M., Ramos, B. P., Paspalas, C. D., Shu, Y., Simen, A., Arnsten, A. F., et al. (2007). Alpha2A-adrenoceptors strengthen working memory networks by inhibiting cAMP-HCN channel signaling in prefrontal cortex. Cell 129, 397-410. doi: 10.1016/j.cell.2007.03.015

$\mathrm{Xu}$, W., and Sudhof, T. C. (2013). A neural circuit for memory specificity and generalization. Science 339, 1290-1295. doi: 10.1126/science.1229534

Yi, F., Danko, T., Botelho, S. C., Patzke, C., Pak, C., Sudhof, T. C., et al. (2016). Autism-associated SHANK3 haploinsufficiency causes Ih channelopathy in human neurons. Science 352:aaf2669. doi: 10.1126/science. aaf2669

Ying, S. W., Tibbs, G. R., Picollo, A., Abbas, S. Y., Sanford, R. L., Goldstein, P. A., et al. (2011). PIP2-mediated HCN3 channel gating is crucial for rhythmic burst firing in thalamic intergeniculate leaflet neurons. J. Neurosci. 31, 10412-10423. doi: 10.1523/JNEUROSCI.0021-11.2011

Conflict of Interest Statement: The authors declare that the research was conducted in the absence of any commercial or financial relationships that could be construed as a potential conflict of interest.

Copyright (c) 2018 Stieglitz, Fenske, Hammelmann, Becirovic, Schöttle, Delorme, Schöll-Weidinger, Mader, Deussing, Wolfer, Seeliger, Albrecht, Wotjak, Biel, Michalakis and Wahl-Schott. This is an open-access article distributed under the terms of the Creative Commons Attribution License (CC BY). The use, distribution or reproduction in other forums is permitted, provided the original author(s) or licensor are credited and that the original publication in this journal is cited, in accordance with accepted academic practice. No use, distribution or reproduction is permitted which does not comply with these terms. 